[3] a) For example, C. G. Someda, "Simple low-loss joints between single-mode optical fibers," Bell Sys. Tech. J., vol. 52, p. 1477, 1973.

b) J. Guttmann, O. Krumholz, and E. Pfeiffer, "Optical fiberstripline-coupler," Appl. Opt., vol. 14, p. 1225, 1975.

[4] A. Arnaud, "Transverse coupling in fiber optics, Part I: Coupling between two trapped modes," Bell Sys. Tech. J., vol. 53, p. 217, 1974.

[5] H. P. Hsu and A. F. Milton, "Single mode optical fiber pickoff coupler," Appl. Opt., vol. 15, p. 2310, 1976.

[6] P. K. Tien, R. J. Martin, and S. Riva-Sanseverino, "Novel metal-clad optical components and method of isolating high index substrates for forming integrated optical circuits," Appl. Phys. Lett., vol. 27, p. 251, 1975.

[7] D. G. Dalgoutte, R. B. Smith, G. Achutaramayya, and J. H. Harris, "Externally mounted fibers for integrated optics interconnections," Appl. Opt., vol. 14, p. 1860, 1975.

[8] A. F. Milton and W. K. Burns, "Tapered velocity couplers for integrated optics: Design," Appl. Opt., vol. 14, p. 1207, 1975.

[9] P. K. Tien, "Light wave in thin films and integrated optics," Appl. Opt., vol. 10, p. 2395, 1971.
[10] W. K. Burns, private communication.

[11] G. B. Hocker and W. K. Burns, "Modes in Diffused Optical Waveguides of Arbitrary Index Profile," IEEE J. Quantum Electron., vol. QE-11, p. 270, 1975.

[12] T. Wojeck, private communication.

[13] D. Marcuse, Light Transmission Optics. ch. 9.

[14] W. K. Burns and A. F. Milton, "Mode Conversion in PlanarDielectric Separating Waveguides," IEEE J. Quantum Electron., vol. QE-11, p. 32, 1975.

[15] P. K. Tien, R. J. Martin, and G. Smolinsky, "Formation of light-guiding interconnections in an integrated optical circuit by composite tapered-film coupling," Appl. Opt., vol. 12, p. 1909, 1973.

[16] R. K. Winn and J. H. Harris, "Beam coupling to linear waveguides using lenslike waveguides," Appl. Opt., vol. 14, p. 1213, 1975.

[17] H. P. Hsu and A. F. Milton, "Flip chip approach to end fire coupling between single-mode optical fibers and channel waveguides," Electron. Lett., vol. 12, p. 404, 1976.

[18] E. A. J. Marcatili, "Dielectric rectangular waveguide and directional coupler for integrated optics," Bell Sys. Tech. J., vol. 48, p. $2071,1969$.

\title{
Periodic Structures for Integrated Optics
}

\author{
AMNON YARIV AND MICHIHARU NAKAMURA
}

\begin{abstract}
This paper deals with the theory and device applications of periodic thin-film waveguides. Topics treated include mode solvtions, optical filters, distributed feedback lasers (DFB), distributed Bragg reflector (DBR) lasers, grating couplers, and phase matching in nonlinear interactions.
\end{abstract}

\section{INTRODUCTION}

$\mathrm{T}$ HE phenomenon of wave propagation in periodic structures or media recurs in many branches of physics and technology. As examples, consider the phenomena of X-ray and electron diffraction in crystals, the diffraction of light from the periodic strain variation accompanying a sound wave, and the band structures and energy gaps of the phonon spectrum and the energy of electrons in a crystal. Among practical devices making use of this phenomenon we may mention the traveling wave tube, the linear particle accelerator, diffraction gratings, and holograms.

Manuscript received October 15, 1976; revised December 12, 1976. This work was supported in part by the National Science Foundation and in part by the Office of Naval Research.

A. Yariv is with the California Institute of Technology, Pasadena, CA 91125.

M. Nakamura is with the Hitachi Central Research Laboratory, Kokubunji, Tokyo 185, Japan.
With the intense pace of recent research in dielectric waveguiding phenomena and devices, a field sometimes referred to as "Integrated Optics," a new generation of optical devices was born, one utilizing periodic thin-film dielectric waveguides. Some of the devices in this family which have already been demonstrated include thin-film optical filters, distributed feedback lasers (DFB), distributed Bragg reflector lasers (DBR), and input and output grating couplers for dielectric waveguides.

This paper attempts to summarize and review the theoretical and experimental progress in periodic thin-film devices with a special effort devoted to bringing out the underlying unifying concepts.

\section{WAveguide Modes}

Before embarking on an analysis of periodic waveguides devices it is important to understand the basic modes which can be supported in a uniform (unperturbed) waveguide. We will limit our attention to slab planar waveguides such as that sketched in Fig. 1. The extension to channel waveguides is straightforward [1], [2] but introduces considerable mathematical complexity without, for the most part, modifying in a qualitative fashion any of the basic phenomena. 


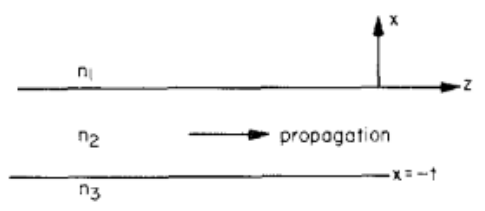

Fig. 1. Slab $\left(\partial / \partial_{y}=0\right)$ dielectric waveguide.

We need to solve the wave equation

$$
\nabla^{2} E(r)+k^{2} n^{2}(r) E(r)=0
$$

where $k^{2} \equiv \omega^{2} \mu \epsilon_{0}=(2 \pi / \lambda)^{2}$ and $n$ is the index of refraction. The solutions are subject to the continuity of the tangential components of $E$ and $H$ at the dielectric interfaces. In (1) the form of the field is taken as

$$
E(r, t)=E(r) \exp i(\omega t-\beta z)
$$

so that it becomes

$$
\left(\frac{\partial^{2}}{\partial x^{2}}+\frac{\partial^{2}}{\partial y^{2}}\right) E(x, y)+\left[k^{2} n^{2}(r)-\beta^{2}\right] E(x, y)=0 .
$$

Putting $\partial / \partial y=0$ in (3) and writing it separately for regions I, II, and III yields:

Region I:

$$
\frac{\partial^{2}}{\partial x^{2}} E(x, y)+\left(k^{2} n_{1}^{2}-\beta^{2}\right) E(x, y)=0
$$

Region II:

$$
\frac{\partial^{2}}{\partial x^{2}} E(x, y)+\left(k^{2} n_{2}^{2}-\beta^{2}\right) E(x, y)=0
$$

Region III:

$$
\frac{\partial^{2}}{\partial x^{2}} E(x, y)+\left(k^{2} n_{3}^{2}-\beta^{2}\right) E(x, y)=0
$$

where $E(x, y)$ is a Cartesian component of $E(x, y)$. Before embarking on a formal solution of (4) we may learn a great deal about the physical nature of the solutions by simple arguments. Let us consider the nature of the solutions as a function of the propagation constant $\beta$ at a fixed frequency $\omega$. Let us assume that $n_{2}>n_{3}>n_{1}$. For $\beta>k n_{2}$ (that is, regime $a$ in Fig. 2) it follows directly from (4) that $(1 / E)\left(\partial^{2} E / \partial x^{2}\right)>$ 0 everywhere, and $E(x)$ is exponential in all three layers (I, II, III) of the waveguides. Because of the need to match both $E(x)$ and its derivatives at the two interfaces, the resulting field distribution is as shown in Fig. 2(a). The field increases without bound away from the waveguide so that the solution is not physically realizable and thus does not correspond to a real wave.

For $k n_{3}<\beta<k n_{2}$, as in points $b$ and $c$, it follows from (4) that the solution is sinusoidal in region II, since $(1 / E)$ $\left(\partial^{2} E / \partial x^{2}\right)<0$, but is exponential in regions I and III. This makes it possible to have a solution $E(x)$ that satisfies the boundary conditions while decaying exponentially in regions I and III. Two such solutions are shown in $b$ and $c$, Fig. 2 . The energy carried by these modes is confined to the vicinity of the guiding layer II, and we will, consequently, refer to them as confined, or guided, modes. From the above discussion it follows that a necessary condition for their existence is that $k n_{1}, k n_{3}<\beta<k n_{2}$ so that confined modes are possible only when $n_{2}>n_{1}, n_{3}$; that is, the inner layer possesses the highest index of refraction.

Mode solutions for $k n_{1}<\beta<k n_{3}$, regime $d$, correspond according to (4) to exponential behavior in region $I$ and to sinusoidal behavior in regions II and III as illustrated in $d$, Fig. 2 . We will refer to these modes as substrate radiation modes. For $0<\beta<k n_{1}$, as in $e$, the solution for $E(x)$ becomes sinusoidal in all three regions. These are the so-called radiation modes of the waveguides.

A solution of (4) subject to the boundary conditions at the interfaces given below shows that while in regimes $d$ and $e, \beta$ is a continuous variable, the values of allowed $\beta$ in the propagation regime $k n_{3}<\beta<k n_{2}$ are discrete. The number of confined modes depends on the width $t$, the frequency, and the indices of refraction $n_{1}, n_{2}, n_{3}$. At a given wavelength the number of confined modes increases from 0 with increasing $t$. At some $t$, the mode $\mathrm{TE}_{1}$ becomes confined. Further increases in $t$ will allow $\mathrm{TE}_{2}$ to exist as well, and so on.

A useful point of view is one of considering the wave propagation in the inner layer 2 as that of a plane wave propagating at some angle $\theta$ to the horizontal axis and undergoing a series of total internal reflections at the interfaces II-I and II-III. This is based on (4b). Assuming a solution in the form of $E \propto \sin (h x+\alpha) \exp (-i \beta z)$, we obtain

$$
\beta^{2}+h^{2}=k^{2} n_{2}^{2} \text {. }
$$

The resulting right-angle triangles with sides $\beta, h$, and $k n_{2}$ are shown in Fig. 2. Note that since the frequency is constant, $k n_{2} \equiv(\omega / c) n_{2}$ is the same for cases $b, c, d$, and $e$. The propagation can thus be considered formally as that of a plane wave along the direction of the hypotenuse with a constant propagation constant $k n_{2}$. As $\beta$ decreases, $\theta$ increases until, at $\beta=k n_{3}$, the wave ceases to be totally internally reflected at the interface III-II. This follows from the fact that the guiding condition $\beta>k n_{3}$ leads, using $\beta=k n_{2} \cos \theta$, to $\theta<$ $\cos ^{-1}\left(n_{3} / n_{2}\right)=\theta_{c}$, where $\theta_{c}$ is the total internal reflection angle at the interface between layers II-III. Since $n_{3}>n_{1}$, total reflection at the II-III interface guarantees total internal reflection at the I-II interface.

We are now ready to derive the mode solutions for the general slab waveguide shown in Fig. 1. We limit the derivation to the guided modes which, according to Fig. 2, have propagation constants $\beta$

$$
k n_{3}<\beta<k n_{2}
$$

where $n_{3}>n_{1}$.

\section{TE Modes}

The TE modes possess field components $E_{y}, H_{x}, H_{z}$ only. The field component $E_{y}$ of the mode obeys the wave equation

$$
\nabla^{2} E_{y i}(x, y, z)+\omega^{2} \mu \epsilon_{0} n_{i}^{2} E_{y i}=0, \quad i=1,2,3
$$

where $i$ refers to the layer, and the (real) electric field is given by

$$
E_{y i}(x, y, z, t)=\operatorname{Re}\left[E_{y i}(x, y, z) e^{i \omega t}\right] .
$$




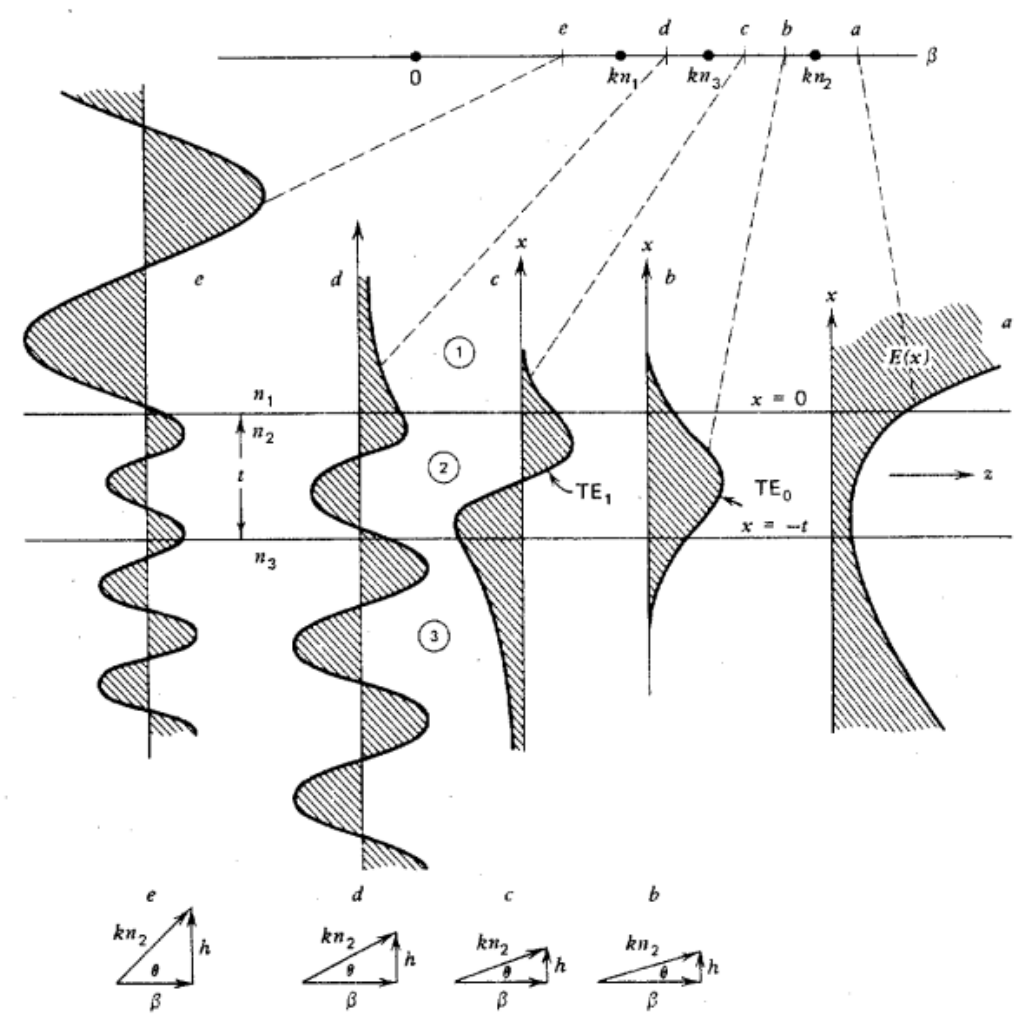

Fig. 2. Top: the different regimes, $a, b, c, d, e$, of the propagation constant, $\beta$, of the waveguide shown in Fig. 1. Middle: the field distributions corresponding to the different values of $\beta$. Bottom: the propagation triangles corresponding to the different propagation regimes.

For waves propagating along the $z$ direction and for $\partial / \partial y=0$ we have

$$
E_{y i}(x, y, z)=\&_{y i}(x) e^{-i \beta z} .
$$

The transverse function $\&_{y i}(x)$ is taken as

$$
\mathscr{E}_{y}=\left[\begin{array}{lc}
C \exp (-q x), & 0 \leqslant x<\infty \\
C[\cos (h x)-(q / h) \sin (h x)], & -t \leqslant x \leqslant 0 \\
C[\cos (h t)+(q / h) \sin (h t) \exp [p(x+t)], & -\infty<x \leqslant-t .
\end{array}\right.
$$

Applying (6) to (8) results in

$$
\begin{aligned}
& h=\left(n_{2}^{2} k^{2}-\beta^{2}\right)^{1 / 2} \\
& q=\left(\beta^{2}-n_{1}^{2} k^{2}\right)^{1 / 2} \\
& p=\left(\beta^{2}-n_{3}^{2} k^{2}\right)^{1 / 2} \\
& k \equiv \omega / c .
\end{aligned}
$$

The acceptable solutions for $\&_{y}$ and $\mathcal{H}_{z}=(i / \omega \mu)\left(\partial \mathscr{E}_{y} / \partial x\right)$ should be continuous at both $x=0$ and $x=-t$. The choice of coefficients in (8) is such as to make \&y continuous at both interfaces as well as $\left(\partial \varepsilon_{y} / \partial x\right)$ at $x=0$. By imposing the continuity requirement on $\partial \varepsilon_{y} / \partial x$ at $x=-t$, we get from (8)

$$
h \sin (h t)-q \cos (h t)=p[\cos (h t)+(q / h) \sin (h t)]
$$

or

$$
\tan (h t)=\frac{p+q}{h\left(1-p q / h^{2}\right)} .
$$

The last equation in conjunction with (9) is used to obtain the eigenvalues $\beta$ for the confined TE modes. An example of such a solution is shown in Fig. 3 .

The constant, $C$, appearing in (8) is arbitrary, yet for many applications, especially those in which propagation and exchange of power involve more than one mode, it is advantageous to define $C$ in such a way that it is simply related to total power in the mode. This point will become clearer below. We choose $C$ so that the field $\varepsilon_{y}(x)$ in (8) corresponds to a power flow of $1 \mathrm{~W}$ (per unit width in the $y$ direction) in the mode. A mode for which $E_{y}=A \varepsilon_{y}(x)$ will thus correspond to a power flow of $|A|^{2} \mathrm{~W} / \mathrm{m}$. The normalization condition becomes

$$
-\frac{1}{2} \int_{-\infty}^{\infty} E_{y} H_{x}^{*} d x=\frac{\beta_{m}}{2 \omega \mu} \int_{-\infty}^{\infty}\left[\varepsilon_{y}^{(m)}(x)\right]^{2} d x=1
$$

when the symbol $m$ denotes the $m$ th confined TE mode [corresponding to the $m$ th eigenvalue of (11)] and $H_{x}=$ $-i\left(\omega \mu_{0}\right)^{-1} \partial E_{y} / \partial z$.

Using (8) in (11) leads, after substantial but straightforward calculation, to

$$
C_{m}=2 h_{m}\left[\frac{\omega \mu}{\left|\beta_{m}\right|\left[\left(t+\left(1 / q_{m}\right)+\left(1 / p_{m}\right)\left(h_{m}^{2}+q_{m}^{2}\right)\right]\right.}\right]^{1 / 2} \text {. }
$$

Since the modes $\varepsilon_{y}^{(m)}$ are orthogonal we have

$$
\int_{-\infty}^{\infty} \varepsilon_{y}^{(l)} \varepsilon_{y}^{(m)} d x=\frac{2 \omega \mu}{\beta_{m}} \delta_{l, m}
$$




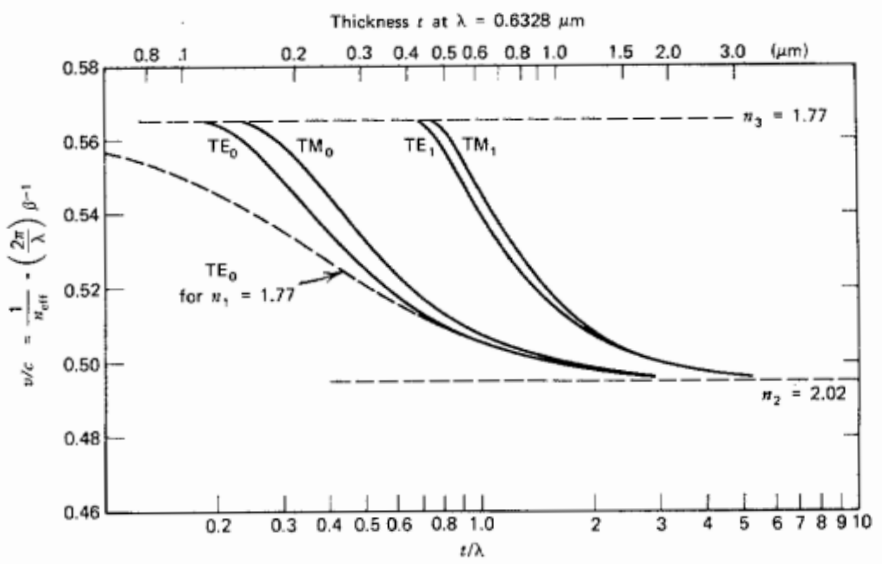

Fig. 3. Dispersion curves for the confined modes of $\mathrm{ZnO}$ on sapphire waveguide $n_{1}=1$.

\section{TM Modes}

The derivation of the confined TM modes is similar in principle to that of the TE modes. The field components are

$$
\begin{aligned}
& H_{y}(x, z, t)=\mathcal{H}_{y}(x) \exp i(\omega t-i \beta z) \\
& E_{x}(x, z, t)=\frac{i}{\omega \epsilon} \frac{\partial H_{y}}{\partial z}=\frac{\beta}{\omega \epsilon} \mathcal{H}_{y}(x) \exp i(\omega t-\beta z) \\
& E_{z}(x, z, t)=-\frac{i}{\omega \epsilon} \frac{\partial H_{y}}{\partial x} .
\end{aligned}
$$

The transverse function, $\mathscr{H}_{y}(x)$, is taken as

$$
\mathscr{H}_{y}(x)= \begin{cases}-C\left[\frac{h}{q} \cos (h t)+\sin (h t)\right] \exp p(x+t), \\ C\left[-\frac{h}{q} \cos (h x)+\sin (h x)\right], & -t<x<0 \\ -\frac{h}{q} C e^{-q x}, & x>0 .\end{cases}
$$

The continuity of $H_{y}$ and $E_{z}$ at the two interfaces leads, in a manner similar to $(10)$, to the eigenvalue equation

$$
\tan (h t)=\frac{h(\bar{p}+\bar{q})}{h^{2}-\bar{p} \bar{q}}
$$

where

$$
\bar{p} \equiv \frac{n_{2}^{2}}{n_{3}^{2}} p \quad \bar{q}=\frac{n_{2}^{2}}{n_{1}^{2}} q .
$$

The normalization constant, $C$, is chosen so that the field represented by (14) and (15) carries one watt per unit width in the $y$ direction

$$
\frac{1}{2} \int_{-\infty}^{\infty} H_{y} E_{x}^{*} d x=\frac{\beta}{2 \omega} \int_{-\infty}^{\infty} \frac{\mathcal{H}_{y}^{2}(x)}{\epsilon(x)} d x=1,
$$

or, using $n_{i}^{2} \equiv \epsilon_{i} / \epsilon_{0}$,

$$
\int_{-\infty}^{\infty} \frac{\left[\mathcal{H}_{y}^{(m)}(x)\right]^{2}}{n^{2}(x)} d x=\frac{2 \omega \epsilon_{0}}{\beta_{m}} .
$$

Carrying out the integration using (15) gives

$$
\begin{aligned}
C_{m} & =2 \sqrt{\frac{\omega \epsilon_{0}}{\beta_{m} t_{\mathrm{eff}}}} \\
t_{\mathrm{eff}} & \equiv \frac{\bar{q}^{2}+h^{2}}{\bar{q}^{2}}\left(\frac{t}{n_{2}^{2}}+\frac{q^{2}+h^{2}}{\bar{q}^{2}+h^{2}} \frac{1}{n_{1}^{2} q}+\frac{p^{2}+h^{2}}{\bar{p}^{2}+h^{2}} \frac{1}{n_{3}^{2} p}\right) .
\end{aligned}
$$

The general properties of the TE and TM mode solutions are illustrated in Fig. 3. In general, a mode becomes confined above a certain (cutoff) value of $t / \lambda$. At the cutoff value $p=0$, the mode extends to $x=-\infty$. For increasing values of $t / \lambda, p>0$, and the mode becomes increasingly confined to layer 2. This is reflected in the effective mode index $2 \pi /(\beta \lambda)$ that, at cutoff, is equal to $n_{3}$, and which, for large $t / \lambda$, approaches $n_{2}$. In a symmetric waveguide $\left(n_{1}=n_{3}\right)$ the lowest order modes $\mathrm{TE}_{0}$ and $\mathrm{TM}_{0}$ have no cutoff and are confined for all values of $t / \lambda$. The selective excitation of waveguide modes by means of prism couplers and a determination of their propagation constants $\beta_{m}$ are described in [3], [4] .

\section{Mode Coupling in Periodic Waveguides}

In this section we will derive the equations describing mode coupling caused by periodic perturbations. Before approaching the formal problem we will consider some of the more general and qualitative aspects of mode coupling.

\section{Coupling Between Modes}

Consider two different spatial modes of the unperturbed waveguide given by

$$
\begin{aligned}
& \boldsymbol{E}_{1}(x, y, z, t)=\boldsymbol{f}_{1}(x, y) \exp \left(-i \beta_{1} z\right) \exp (i \omega t) \\
& \boldsymbol{E}_{2}(x, y, z, t)=\boldsymbol{f}_{2}(x, y) \exp \left(-i \beta_{2} z\right) \exp (i \omega t) .
\end{aligned}
$$

Let the effect of the perturbation be represented by $\Delta n^{2}(x$, $y, z)$ which is defined as the difference between the squared index of refraction of the periodic waveguide minus that of the uniform waveguide. In the case of a corrugated wave $\Delta n^{2}(x, y, z)$ is thus the spatial function whose value at each point $(x, y, z)$ is equal to the change in $n^{2}$ caused by the corrugation.

It follows from the basic relation between the dielectric constant $\epsilon$ and the index $n$ which is $\epsilon=\epsilon_{0} n^{2}$, and the relation

$$
\boldsymbol{P}=\left(\epsilon-\epsilon_{0}\right) \boldsymbol{E}=\epsilon_{0}\left(n^{2}-1\right) \boldsymbol{E}
$$

between the electric field $E$ and the medium polarization (dipole moment per unit volume) $P$ that the field $E_{1}(x, y$, $z, t$ ) propagating in the perturbed (i.e., periodic) waveguide will give rise to a new perturbation polarization

$$
\boldsymbol{P}_{\text {pert }}=\epsilon_{0} \Delta n^{2}(x, y, z) E_{1}(x, y, z, t) .
$$

Now if this field, acting as a distributed source, can feed power into (or out of) mode $\boldsymbol{E}_{2}(x, y, z, t)$, then we say that the perturbation $\Delta n^{2}(x, y, z)$ couples (i.e., causes power exchange) between modes $E_{1}$ and $E_{2}$. Let us find, next, under what conditions this coupling takes place.

The power per unit volume flowing into the field $E_{2}$ from the polarization source $\boldsymbol{P}_{\text {pert }}$ is

$$
P_{1 \rightarrow 2 / \mathrm{vol}}=E_{2}(x, y, z, t) \cdot \frac{\partial}{\partial t} P_{\text {pert }}(x, y, z, t) ;
$$


averaging the power flow over one optical period $T=2 \pi / \omega$ we obtain, using (19) in (22),

$$
\begin{aligned}
P_{1 \rightarrow 2 / \mathrm{vol}}= & \frac{\omega}{2 \pi} \int_{0}^{2 \pi / \omega} P_{1 \rightarrow 2}(t) / \mathrm{vol} d t \\
= & \frac{\omega}{2 \pi} \int_{0}^{2 \pi / \omega}\left\{\frac { 1 } { 4 } \left(f_{2}(x, y) \exp i\left(\omega t-\beta_{2} z\right)\right.\right. \\
& + \text { c. c. }) \cdot \frac{\partial}{\partial t}\left[\epsilon_{0} \Delta n^{2}(x, y, z) f_{1}(x, y)\right. \\
& \left.\left.\cdot \exp i\left(\omega t-\beta_{1} z\right)+\text { c.c. }\right)\right\} d t \\
= & \frac{\omega \epsilon_{0} \Delta n^{2}(x, y, z)}{2} \operatorname{Im}\left[f_{2}^{*} \cdot f_{1} \exp i\left(\beta_{2}-\beta_{1}\right) z\right] .
\end{aligned}
$$

The source of this power flow is, of course, the power of mode 1. It is not surprising that a similar calculation for the power flow into mode 1 (here we take $\Delta \boldsymbol{P}=\epsilon_{0} \Delta n^{2} E_{2}$ ) yields

$$
P_{2 \rightarrow 1 / \mathrm{vol}}=-P_{1 \rightarrow 2 / \mathrm{vol}},
$$

so that, in the absence of gain or losses, the total power is conserved. Power fed via the perturbation into mode 1 originates in mode 2 and vice versa.

The total power flowing into mode 2 from mode 1 is obtained by integrating the power density as given by (24) over all space

$$
\begin{aligned}
P_{1 \rightarrow 2} & =\int_{\text {space }} \frac{\omega \epsilon_{0} \Delta n^{2}(x, y, z)}{2} \operatorname{Im}\left[\boldsymbol{f}_{2}^{*} \cdot \boldsymbol{f}_{1}\right. \\
& \left.\cdot \exp i\left(\beta_{2}-\beta_{1}\right) z\right] d x d y d z .
\end{aligned}
$$

In the periodic structures which are considered in this article the direction of periodicity is taken along the direction of propagation $z$. Since $\Delta n^{2}(x, y, z)$ is periodic in $z$ we can expand it in general as a Fourier series

$$
\Delta n^{2}(x, y, z)=\sum_{l=-\infty} a_{l}(x, y) \exp \left(i l \frac{2 \pi}{\Lambda} z\right)
$$

where $\Lambda$ is the period. By substituting this expression into (26) and integrating over a distance $z$ large compared to $\Lambda$ we find that a necessary condition for power flow, i.e., for nonvanishing of the integral is that

$$
\int_{L>>\Lambda} \exp \left[i\left(\beta_{2}-\beta_{1}+l \frac{2 \pi}{\Lambda}\right) z\right] d z \neq 0
$$

for some integer $l$. This happens when

$$
\beta_{1}-\beta_{2}=l \frac{2 \pi}{\Lambda} .
$$

Condition (29) is of fundamental importance and we will refer to it as "longitudinal phase matching" or just as phase matching. We note that the coupling between modes 1 and 2 is brought about by the $l$ th Fourier harmonic of the spatial perturbation (27). It would thus follow naturally that the strength of this coupling will depend on $a_{l}$ and will vanish al together when $a_{l}=0$. This situation is analogous to the case in X-ray diffraction where certain diffraction orders may be missing in a given crystal class although the appropriate Bragg condition [equivalent to our condition (29)] is satisfied.

The second condition necessary to ensure power flow $P_{1 \rightarrow 2}$ is that the transverse part of the integral (26) be nonvanishing, i.e.,

$$
\int_{\substack{\text { cross } \\ \text { section }}} \Delta n^{2}(x, y, z) f_{1}^{*}(x, y) \cdot f_{2}(x, y) d x d y \neq 0
$$

The integral (30) is referred to as the transverse overlap integral and the integrand is essentially the product of the transverse profiles of the two interacting modes and the perturbation which couples them. If the phase matching is achieved via the $l$ th space harmonic (i.e., (29) is satisfied) then the relevant part of $(30)$ is

$$
\int_{\substack{\text { cross } \\ \text { section }}} a_{l}(x, y) f_{1}^{*} \cdot \boldsymbol{f}_{2}(x, y) d x d y \neq 0,
$$

i.e., only the transverse field distribution of the phase-matched harmonic $(l)$ figures in the overlap integral.

A number of situations occur in practice which may require a slight generalization of condition (31). These involve a periodic index perturbation due to the photoelastic, electrooptic, or magnetooptic effects. In this case condition (31) is replaced by an obvious tensorial extension [5]. Another interesting case is one in which the perturbation is periodic in time as well as in space. In this case the requi,ement that the time-averaged power flow be nonvanishing is satisfied only when the modes' frequencies $\omega_{1}-\omega_{2}$ satisfy $\omega_{1}-\omega_{2}=$ $\pm \Omega$ where $\Omega$ is the frequency of the perturbation.

The general properties of mode coupling considered above are of great importance in the sense that they can be used to determine what processes can take place and what kind of perturbation is needed to couple a given pair of modes. We will demonstrate this point by a number of examples.

Let us assume that we need to couple together a forward guided mode with a spatial propagation factor $\exp (-i \beta z)$ to a backward wave of the same mode description, i.e., one propagating as $\exp (i \beta z)$. It follows from (29) that the spatial perturbation period $\Lambda$ needs to satisfy

$$
\beta_{1}-\beta_{2}=2 \beta=l \frac{2 \pi}{\Lambda},
$$

or since $\beta=2 \pi / \lambda_{g}$, where $\lambda_{g}$ is the mode wavelength in the guide,

$$
\Lambda=l \frac{\lambda_{g}}{2} \equiv l \frac{\lambda}{2 n_{\mathrm{eff}}},
$$

where $l=1,2, \cdots$ is some integer. As noted above, condition (33) is necessary but not sufficient. As a matter of fact we will consider later in this article a case of a symmetric square wave corrugation where the second-order coupling $(l=2)$ vanishes, since $a_{2}=0$. 
A phase-matching diagram illustrating condition (32) is shown in Fig. 4(a).

As a second example consider the problem of designing a grating coupler [6] for coupling from a guided confined mode with a propagation constant $\beta$ to a radiation mode (i.e., a mode of type $e$ in Fig. 2) which escapes at an angle $\theta$ into the semi-infinite upper layer with index $n_{1}$. The situation is illustrated in Fig. 4(b). The wave in medium 1 has the form of

$$
E_{y} \propto \exp \left[-i \frac{\omega n_{1}}{c}(z \cos \theta+x \sin \theta)\right]
$$

as appropriate to a plane wave with a wavelength $\lambda / n_{1}$ propagating at an angle $\theta$ to the $z$ axis.

The grating, which may be corrugated into one of the two interfaces must, according to (29), have a period $\Lambda$ satisfying

$$
\beta-\frac{\omega n_{1}}{c} \cos \theta=l \frac{2 \pi}{\Lambda}, \quad l= \pm 1, \pm 2, \cdots .
$$

Using the relation $\beta=2 \pi / \lambda_{g}$ and $\lambda=c / f(\lambda$ is the free-space wavelength, $f$ is the frequency) we can rewrite (35) in a somewhat more convenient form

$$
\frac{1}{\lambda_{g}}-\frac{n_{1}}{\lambda} \cos \theta=\frac{l}{\Lambda} \text {. }
$$

As a third example, which is not related to waveguiding phenomena but demonstrates the power of the general principles derived above, let us consider the problem of the planar diffraction grating as illustrated in Fig. 4(c).

An optical plane wave is incident at angle $\theta_{1}$ on a grating of period $\Lambda$. We need to find the reflection angle $\theta_{2}$. We apply the longitudinal phase-matching condition (29) replacing the longitudinal propagation constant $\beta$ by $k \cos \theta$. The result is

$$
k \cos \theta_{1}-k \cos \theta_{2}=l \frac{2 \pi}{\Lambda}
$$

or using $k=\omega / c=2 \pi / \lambda$ (since in air $n=1)$

$$
\frac{1}{\lambda}\left(\cos \theta_{1}-\cos \theta_{2}\right)=\frac{l}{\Lambda} .
$$

The different integers $l=0, \pm 1, \pm 2$ for which $\left|\cos \theta_{2}\right|<1$ correspond to the various orders of the grating. Anybody who had occasion to derive the grating equation (37) using path difference arguments will appreciate the simplicity of the phase-matching approach.

\section{The Coupled-Mode Equations}

Having set the stage by deriving in Section II the waveguide modes and describing in the early part of this section the qualitative nature of mode coupling, we derive next the coupled-mode formalism for treating mode coupling by a periodic index perturbation.

The physical situation is that of a slab dielectric waveguide whose spatial distribution of index of refraction is $n^{2}(r)$. The waveguide is next perturbed by a spatial periodic index modulation $\Delta n^{2}(x, y, z)$. Maxwell's propagation equation in

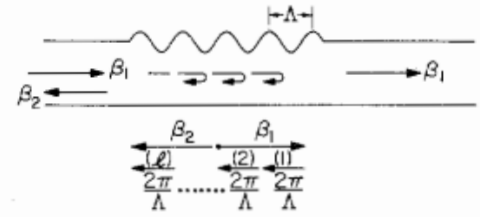

(a)
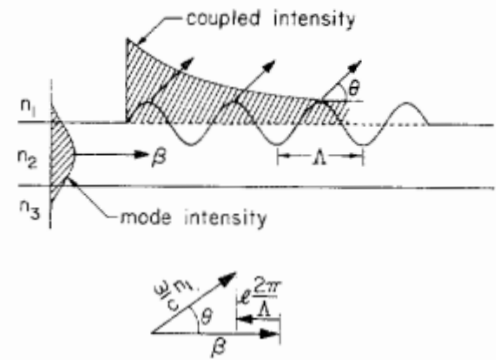

(b)

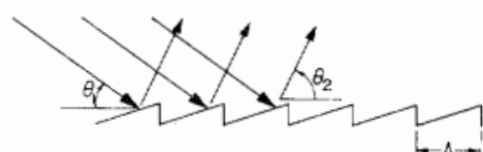

$\underset{\frac{\omega}{c} \cos \theta_{2}}{\stackrel{\frac{\omega}{c} \cos \theta_{1}}{\ell \frac{2 \pi}{\Lambda}}}$

(c)

Fig. 4. Longitudinal phase matching diagram illustrating the application of (29) to a number of periodic devices. (a) Corrugated waveguide reflector. (b) Corrugated waveguide output coupler. (c) Diffraction grating.

such a waveguide is

$$
\nabla^{2} E(\bar{r}, t)=\mu \epsilon_{0} \frac{\partial^{2} \boldsymbol{E}}{\partial t^{2}}+\mu \frac{\partial^{2}}{\partial t^{2}} \boldsymbol{P}(r, t)
$$

where $P$, the total polarization vector in the medium, can be written as

$$
P(r, t)=\epsilon_{0}\left[n^{2}(r)+\Delta n^{2}(r)-1\right] E(r, t) .
$$

Using (39) in (38) and recognizing that $\epsilon_{0} n^{2}(r)$ is the dielectric constant $\epsilon(r)$ of the unperturbed waveguide we arrive at

$$
\nabla^{2} E_{y}-\mu \epsilon(r) \frac{\partial^{2} E_{y}}{\partial t^{2}}=\mu \epsilon_{0} \frac{\partial^{2}}{\partial t^{2}} \Delta n^{2}(r) E_{y}(r, t)
$$

and similar equations for $H_{x}$ and $H_{z}$.

Limiting our attention to coupling between confined modes only (i.e., not involving the radiation modes type $d$ and $e$ in Fig. 2) we expand the total field $E_{y}(r, t)$ in (40) as

$$
E_{y}(r, t)=\frac{1}{2} \sum_{m} A_{m}(z) \mathscr{E}_{y}^{(m)}(x) \exp i\left(\omega t-\beta_{m} z\right)+\text { c.c. }
$$

where $m$ indicates the $m$ th discrete eigenmode of (40), which satisfies

$$
\left(\frac{\partial^{2}}{\partial x^{2}}-\beta_{m}^{2}\right) \varepsilon_{y}^{(m)}(r)+\omega^{2} \mu \epsilon(r) \varepsilon_{y}^{(m)}(r)=0 .
$$

Substitution of (41) in (40) and using $\nabla^{2}=-\beta^{2}+\partial^{2} / \partial x^{2}$ 
leads to

$$
\begin{aligned}
e^{i \omega t} & \sum_{m}\left[\frac { A _ { m } } { 2 } \left(-\beta_{m}^{2} \varepsilon_{y}^{(m)}+\frac{\partial^{2} \varepsilon_{y}^{(m)}}{\partial x^{2}}\right.\right. \\
& \left.+\omega^{2} \mu \epsilon(r) \varepsilon_{y}^{(m)}\right) e^{-i \beta_{m} z} \\
& \left.+\frac{1}{2}\left(-2 i \beta_{m} \frac{d A_{m}}{d z}+\frac{d^{2} A_{m}}{d z^{2}}\right) \varepsilon_{y}^{(m)} e^{-i \beta_{m} z}\right]+ \text { c.c. } \\
& =\mu \frac{\partial^{2}}{\partial t^{2}}\left(\epsilon_{0} \Delta n^{2} E_{y}(\boldsymbol{r}, t)\right) .
\end{aligned}
$$

First we note that in view of (42), the sum of the first three terms in (43) is zero. We assume "slow" variation so that

$$
\left|\frac{d^{2} A_{m}}{d z^{2}}\right|<<\beta_{m}\left|\frac{d A_{m}}{d z}\right|
$$

and obtain from (43)

$$
\begin{aligned}
\sum_{m} & -i \beta_{m} \frac{d A_{m}}{d z} \varepsilon_{y}^{(m)} \exp i\left(\omega t-\beta_{m} z\right)+\text { c.c. } \\
& =-\omega^{2} \mu \epsilon_{0} \Delta n^{2} \cdot E_{y}(\boldsymbol{r}, t) .
\end{aligned}
$$

We next take the product of (44) with $\varepsilon_{y}^{(s)}(x)$ and integrate from $-\infty$ to $\infty$. The result, using (13) is

$$
\begin{gathered}
\frac{d A_{s}^{(-)}}{d z} \exp i\left(\omega t+\beta_{s} z\right)-\frac{d A_{s}^{(+)}}{d z} \exp i\left(\omega t-\beta_{s} z\right)-\text { c.c. } \\
=\frac{i \omega \epsilon_{0}}{2} \int_{-\infty}^{\infty} \Delta n^{2}(x, z) E_{y}(x, z, t) छ_{y}^{(s)}(x) d x
\end{gathered}
$$

where we recall that the summation over $m$ in (44) contains two terms involving $\varepsilon_{y}^{(m)}(x)$ for each value of $m$-one, designated as (-), traveling in the $-z$ direction, and the other $(+)$, traveling in the $+z$ direction.

Equation (45) is the starting point for the treatment of mode coupling in periodic waveguides.

\section{The Coupled-Mode Equations for Periodic Waveguides}

Consider a dielectric wave with a corrugated interface such as shown in Fig. 5. The deviation of the spatial distribution of $n^{2}(r)$ due to the corrugation from that of the uniform waveguide is represented by $\Delta n^{2}(x, z)$ in (45). If we expand $E_{y}(x, z, t)$ in (45) in terms of waveguide modes

$$
E_{y}(x, z, t)=\sum_{m} \frac{A_{m}}{2} \mathscr{E}_{y}^{(m)}(x) \exp i\left(\omega t-\beta_{m} z\right)+\text { c.c. },
$$

we obtain

$$
\begin{aligned}
& \frac{d A_{s}^{(-)}}{d z} \exp i\left(\omega t+\beta_{s} z\right)-\frac{d A_{s}^{(+)}}{d z} \exp i\left(\omega t-\beta_{s} z\right)-\text { c.c. } \\
& \quad=\frac{i \omega \epsilon_{0}}{4} \sum_{m}\left[A_{m} \int_{-\infty}^{\infty} \Delta n^{2}(x, z) \varepsilon_{y}^{(m)}(x) \xi_{y}^{(s)}(x) d x\right. \\
& \left.\quad \cdot \exp i\left(\omega t-\beta_{m} z\right)+\text { c.c. }\right] .
\end{aligned}
$$

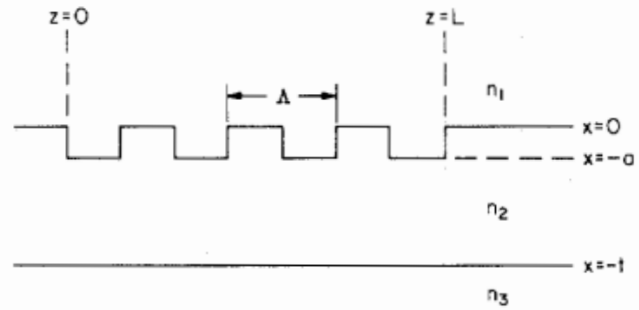

Fig. 5. Corrugated periodic waveguide.

We may consider the right side of (46) as a source term driving the forward wave $A_{s}^{(+)} \exp \left[i\left(\omega t-\beta_{s} z\right)\right]$ and the backward wave $A_{s}^{(-)} \exp \left[i\left(\omega t+\beta_{s} z\right)\right]$. In order for a wave to be driven by a source it is necessary that both the wave and the source have the same frequency so that the interaction will not average out to zero over a long time (long compared to a period of their difference frequency). Since in our case $\Delta n^{2}(x, z)$ does not depend on $t$ this condition is satisfied. Equally important: both source and term must have nearly the same phase dependence $\exp (i \beta z)$ so that the interaction does not average out to zero with distance of propagation $z$. If, for example, it is desired that the forward wave $A_{s}^{(+)} \exp [i(\omega t-$ $\left.\beta_{s} z\right)$ ] be excited, it is necessary that at least one term on the right side of (46), say the $l$ th one, vary as exp $[i(\omega t-\beta z)]$ with $\beta \approx \beta_{s}$. If no other terms on the right side of (46) satisfy this condition, we simplify the equation by keeping only the $l$ th on the right. We describe this situation by saying that the perturbation $\Delta n^{2}(x, z)$ couples the forward $(+s)$ mode to the $l$ th mode and vice versa.

To be specific, let us assume that the period $\Lambda$ of the perturbation $n^{2}(x, z)$ is so chosen that $l \pi / \Lambda \approx \beta_{s}$ for some integer l. We can expand $\Delta n^{2}(x, z)$ as

$$
\begin{aligned}
& \Delta n^{2}(x, z)=\Delta n^{2}(x) \sum_{-\infty} a_{q} \exp [i(2 q \pi / \Lambda) z] \\
& \Delta n^{2}(x, z)=\sum_{-\infty}^{\infty} a_{q}(x) \exp \left(i q \frac{2 \pi}{\Lambda} z\right)
\end{aligned}
$$

The right side of (46) now contains a term $(q=1, m=\mathrm{s})$ proportional to $A_{s}^{(+)} \exp \left[i\left(2 l \pi / \Lambda-\beta_{s}\right) z\right]$. But $\Lambda$ was chosen above such that

$$
\frac{2 l \pi}{\Lambda}-\beta_{s} \approx \beta_{s}
$$

so that this term is capable of driving synchronously the amplitude $A_{s}^{(-)} \exp \left(i \beta_{s} z\right)$ on the left side of (46) with the result

$$
\begin{aligned}
\frac{d A_{s}^{(-)}}{d z}= & \frac{i \omega \epsilon_{0}}{4} A_{s}^{(+)} \int_{-\infty}^{\infty} a_{l}(x)\left[\varepsilon _ { v } ^ { ( s ) } \left(y^{\prime} \cdot d x\right.\right. \\
& \cdot \exp i\left[(2 l \pi / \Lambda)-2 \beta_{s}\right] z .
\end{aligned}
$$

The coupling between the backward $A_{s}^{(-)}$and the forward $A_{s}^{(+)}$by the harmonic $a_{l}(x)$ of the corrugation can thus be described by

$$
\frac{d A_{s}^{(-)}}{d z}=\kappa A_{s}^{(+)} \exp [-i 2(\Delta \beta) z]
$$


and, reciprocally,

$$
\frac{d A_{s}^{(+)}}{d z}=\kappa^{*} A_{s}^{(-)} \exp [i 2(\Delta \beta) z]
$$

where

$$
\begin{aligned}
\kappa & =\frac{i \omega \epsilon_{0}}{4} \int_{-\infty}^{\infty} a_{l}(x)\left[\varepsilon_{y}^{(s)}(x)\right]^{2} d x \\
\Delta \beta & \equiv \beta_{s}-\frac{l \pi}{\Lambda} \equiv \beta_{s}-\beta_{0} .
\end{aligned}
$$

We note that the total power carried by both modes is conserved since

$$
\frac{d}{d z}\left[\left|A_{s}^{(-)}\right|^{2}-\left|A_{s}^{(+)}\right|^{2}\right]=0 .
$$

Let us consider the specific "square wave" corrugation of Fig. 5. In this case the periodicity (period $=\Lambda$ ) in the $z$ direction is accounted for by taking

$$
\begin{gathered}
\Delta n^{2}(x, z)=\sum_{-\infty}^{\infty} a_{l}(x) \exp \left(i \frac{2 \pi l}{\Lambda} z\right)=\Delta n^{2}(x)\left[\frac{1}{2}+\frac{2}{\pi}(\sin \eta z\right. \\
\left.\left.+\frac{1}{3} \sin 3 \eta z+\cdots\right)\right]
\end{gathered}
$$

where

$$
\begin{gathered}
\Delta n^{2}(x)= \begin{cases}n_{2}^{2}-n_{1}^{2}, & -a \leqslant x \leqslant 0 \\
0, & \text { elsewhere }\end{cases} \\
\eta \equiv 2 \pi / \Lambda .
\end{gathered}
$$

For even $l, a_{l}$ is zero. For $l$ odd we obtain from (51) and (54)

$$
\kappa=\frac{-\omega \epsilon_{0}}{4 \pi l} \int_{-\infty}^{\infty} \Delta n^{2}(x)\left[\mathscr{E}_{y}^{(s)}(x)\right]^{2} d x .
$$

In practice the period $\Lambda$ is chosen so that, for some particular $l, \Delta \beta \approx 0$. We note that for $\Delta \beta=0$

$$
\Lambda=l \frac{\lambda_{g}^{(s)}}{2}
$$

where $\lambda_{g}^{(s)}=2 \pi / \beta_{s}$ is the guide wavelength of the $s$ th mode.

We can now use the field expansion (8) plus (55) to perform the integration of (56)

$$
\begin{aligned}
\int_{-\infty}^{\infty} \Delta n^{2}(x)\left[\varepsilon_{y}^{(s)}(x)\right]^{2} d x & =\left(n_{2}^{2}-n_{1}^{2}\right) \int_{-a}^{0}\left[\varepsilon_{y}^{(s)}(x)\right]^{2} d x \\
= & \left(n_{2}^{2}-n_{1}^{2}\right) C_{s}^{2} \int_{-a}^{0}\left[\cos \left(h_{s} x\right)\right. \\
& \left.-\frac{q_{s}}{h_{s}} \sin \left(h_{s} x\right)\right]^{2} d x
\end{aligned}
$$

Although the integral can be calculated exactly using (8) and (10), an especially simple result follows if we consider that operation is sufficiently above propagation cutoff, $t\left(n_{2}-\right.$ $\left.n_{3}\right) / s \lambda>>1$ so that from (9) and (10)

$$
\begin{aligned}
& \beta_{s} \approx n_{2} k \\
& h_{s} \rightarrow \frac{\pi s}{t}, \quad s=1,2, \cdots=\text { transverse mode number } \\
& \frac{q_{s}}{h_{s}} \approx\left(n_{2}^{2}-n_{1}^{2}\right)^{1 / 2}\left(\frac{2 t}{s \lambda}\right) .
\end{aligned}
$$

In addition, since $q_{s}>>h_{s}$, we have, from (12)

$$
C_{s}^{2}=\frac{4 h_{s}^{2} \omega \mu}{\beta_{s} t q_{s}^{2}}
$$

in the well-confined regime, and for $h_{s} a<<1$ the integral (58) becomes

$$
\begin{aligned}
\left(n_{2}^{2}-n_{1}^{2}\right) \int_{-a}^{0}\left[\varepsilon_{y}^{(s)}(x)\right]^{2} d x= & \left(n_{2}^{2}-n_{1}^{2}\right) \frac{4 \pi^{2} \omega \mu}{3 n_{2} k_{0}}\left(\frac{a}{t}\right)^{3} \\
& \left(1+\frac{3}{q_{s} a}+\frac{3}{q_{s}^{2} a^{2}}\right),
\end{aligned}
$$

and, using (56),

$$
\begin{aligned}
\kappa_{s} \approx & \frac{2 \pi^{2} s^{2}}{3 l \lambda} \frac{\left(n_{2}^{2}-n_{1}^{2}\right)}{n_{2}}\left(\frac{a}{t}\right)^{3}\left[1+\frac{3}{2 \pi} \frac{\lambda / a}{\left(n_{2}^{2}-n_{1}^{2}\right)^{1 / 2}}\right. \\
& \left.+\frac{3}{4 \pi^{2}} \frac{(\lambda / a)^{2}}{\left(n_{2}^{2}-n_{1}^{2}\right)}\right] .
\end{aligned}
$$

The complicated physical problem of mode coupling in a corrugated waveguide has thus been reduced to a pair of coupled differential equations (50) and an expression (60) for the coupling constant.

\section{Coupled-Mode Solutions}

Let us return to the coupled-mode equations (50). For simplicity let us put the amplitude of the reflected mode $A_{s}^{(-)} \equiv A, A_{s}^{(+)} \equiv B$ and write them as

$$
\begin{aligned}
& \frac{d A}{d z}=\kappa_{a b} B \exp [-i 2(\Delta \beta) z] \\
& \frac{d B}{d z}=\kappa_{a b}^{*} A \exp [+i 2(\Delta \beta) z] .
\end{aligned}
$$

Consider a waveguide with a corrugated section of length $L$ as in Fig. 6. A wave with an amplitude $B(0)$ is incident from the left on the corrugated section.

The solution of $(61)$ in this case subject to $A(L)=0$, i.e., no reflected wave at the output of the periodic section, is [7]

$$
\begin{aligned}
& A(z) e^{i \beta z}= B(0) \frac{i \kappa_{a b} \exp \left(i \beta_{0} z\right)}{-\Delta \beta \sinh (S L)+i S \cosh (S L)} \\
& \cdot \sinh [S(z-L)] \\
& B(z) e^{-i \beta z}= B(0) \frac{\exp \left(-i \beta_{0} z\right)}{-\Delta \beta \sinh (S L)+i S \cosh (S L)} \\
&\{\Delta \beta \sinh [S(z-L)]+i S \cosh [S(z-L)\}
\end{aligned}
$$




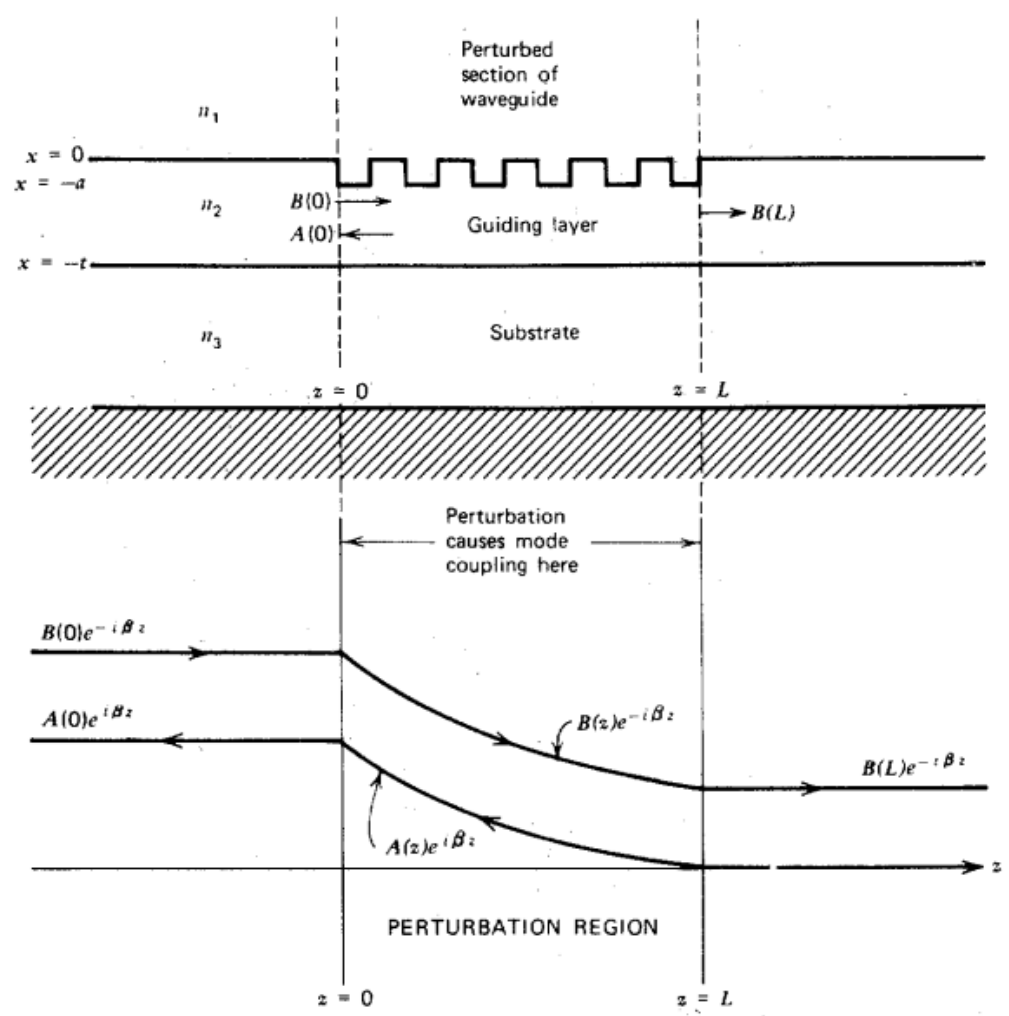

Fig. 6. Upper: Corrugated section of a dielectric waveguide. Lower: Incident and reflected fields.

where

$$
\begin{aligned}
& S=\sqrt{\kappa^{2}-(\Delta \beta)^{2}} \\
& \kappa \equiv\left|\kappa_{a b}\right| .
\end{aligned}
$$

Under phase-matching conditions $\Delta \beta=0$ we have

$$
\begin{aligned}
& A(z)=B(0)\left(\frac{\kappa_{a b}}{\kappa}\right) \frac{\sinh [\kappa(z-L)]}{\cosh (\kappa L)} \\
& B(z)=B(0) \frac{\cosh [\kappa(z-L)]}{\cosh (\kappa L)} .
\end{aligned}
$$

A plot of the mode powers $|B(z)|^{2}$ and $|A(z)|^{2}$ for this case is shown in Fig. 6. For sufficiently large arguments of the hyperbolic cosine and hyperbolic sine functions in (64), the incident mode power drops off exponentially along the perturbation region. This behavior, however, is due, not to absorption, but to reflection of power into the backward traveling mode, $A$, as shown in the figure.

From (41) and (62) we find that the $z$-dependent part of the wave solutions in the periodic waveguide is an exponential with propagation constant

$$
\beta^{\prime}=\beta_{0} \pm i S=\frac{l \pi}{\Lambda} \pm i \sqrt{\kappa^{2}-\left[\beta(\omega)-\beta_{0}\right]^{2}}
$$

where we used $\Delta \beta \equiv \beta-\beta_{0}, \beta_{0} \equiv \pi l / \Lambda$.

We note that for a range of frequencies such that $\Delta \beta(\omega)<$ $\kappa, \beta^{\prime}$ has an imaginary part. This is the so-called "forbidden" region in which the evanescence behavior shown in Fig. 6 occurs and which is formally analogous to the energy gap in semiconductors where the periodic crystal potential causes the electron propagation constants to become complex. Note that for each value of $l, l=1,2,3, \cdots$, there exists a gap whose center frequency $\omega_{0}$ satisfies $\beta\left(\omega_{01}\right)=l \pi / \Lambda$. The exceptions are values of $l$ for which $\kappa$ is zero. Returning to (65) and approximating $\beta(\omega)$ near its Bragg value $(\pi l / \Lambda)$ by $\beta(\omega) \approx$ $(\omega / c) n_{\text {eff }}$, where $n_{\text {eff }}$ is an effective index of refraction, we have

$$
\beta^{\prime} \cong \frac{l \pi}{\Lambda} \pm i\left[\kappa^{2}-\left(\frac{n_{\mathrm{eff}}}{c}\right)^{2}\left(\omega-\omega_{0}\right)^{2}\right]^{1 / 2}
$$

where $\omega_{0}$, the midgap frequency, is the value of $\omega$ for which the unperturbed $\beta$ is equal to $\beta_{0} \equiv l \pi / \Lambda$.

A plot of $\operatorname{Re} \beta^{\prime}$ and $\operatorname{Im} \beta^{\prime}$ for $(l=1)$ versus $\omega$, based on (66), is shown in Fig. 7. We note that the height of the "forbidden" frequency zone is

$$
(\Delta \omega)_{\text {gap }}=\frac{2 \kappa c}{n_{\text {eff }}}
$$

where $\kappa$ is, according to (61), a function of the order $l$. It follows from (65) that

$$
\left(\operatorname{Im} \beta^{\prime}\right)_{\max }=\kappa=\text { coupling coefficient. }
$$

A short section of a corrugated waveguide thus acts as a high-reflectivity mirror for frequencies near the Bragg value, $\omega_{0}$.

Having derived the waveguide modes (Section II), and the coupling formalism (Section III), we are now ready to apply them to some specific cases. 


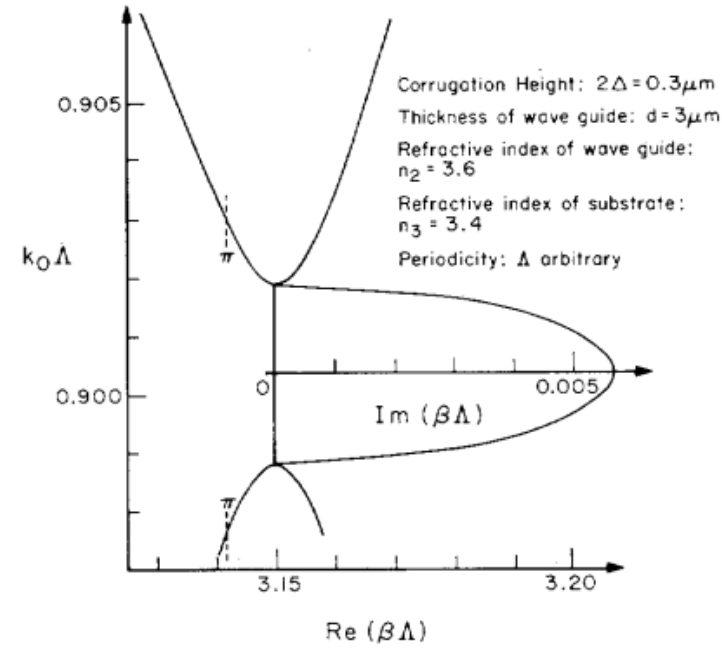

Fig. 7. The dispersion characteristics, i.e., the real and imaginary parts of the propagation constant $\beta$ as a function of frequency in a periodic waveguide.

\section{Fabrication of Surface Corrugations}

The periods of surface corrugations of interest in optical devices vary from as low as $\sim 0.12 \mu \mathrm{m}$ for GaAs DFB lasers to tens of microns for phase matching of 10.6- $\mu \mathrm{m}$ second harmonic generation in GaAs. For corrugation periods longer than of the order of a few microns, conventional photolithography techniques are used for making a photoresist mask. For corrugation periods shorter than $\sim 1 \mu \mathrm{m}$, grating masks are usually produced by laser holographic techniques [6], [8]-[11] , or by electron beam lithography [12]. In the laser holographic techniques, the surface of the guiding layer is spin-coated with a photosensitive resist material (typically, Shipley $1350,1350 \mathrm{~J})$. Argon laser $(4580-\AA$ line) or $\mathrm{He}-\mathrm{Cd}$ laser (4561- $\AA$ or $3250-\AA$ line) beams from a common source are projected onto the photoresist at an angle $\alpha$ as shown in Fig. 8. The interference of these two beams causes an exposure with a sinusoidal intensity variation across the surface with a period of $\Lambda=\lambda_{l} / 2 \sin \alpha$ where $\lambda_{l}$ is the laser wavelength. By adjusting the exposure and development parameters a photoresist grating mask remains on the surface of the substrate [Fig. 5]. The shortest period achievable by this method is $\lambda_{l} / 2$. A further reduction of the corrugation period is obtained by exposing the photoresist in a high-index material [13], [14], as shown in Fig. 9. In this method, laser beams are projected through a high-quality quartz prism. In this case the grating period is $\Lambda=\lambda_{l} / 2 n_{p} \sin \alpha^{\prime}$, where $n_{p}$ is the refractive index of the prism material $\left(n_{p} \approx 1.51\right.$ for quartz), and $\alpha^{\prime}$ is the internal incidence angle. For $\alpha$ near $90^{\circ}$, periods approaching $0.11 \mu \mathrm{m}$ can be produced using a $3250-\AA$ $\mathrm{He}-\mathrm{Cd}$ laser. In the method illustrated by Fig. 9, index matching oil such as xylene was used between the prism and the photoresist layer to increase the optical exposure.

The substrate surface can be corrugated by ion milling or by chemical etching through the photoresist mask as described above. In the ion milling, a broad-area argon ion beam is used to etch grooves into the substrate [8], [15]. The argon ions are accelerated at $0.6-5 \mathrm{keV}$. The milling process occurs essentially through the transfer of momentum by the impinging ions to atoms on the surface of the material. The ion

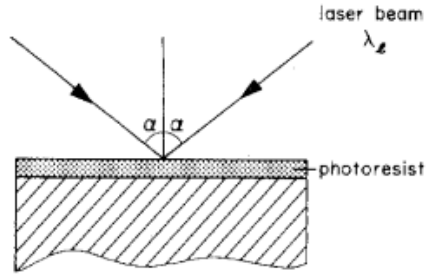

(a)

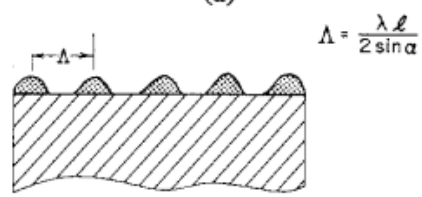

(b)

Fig. 8. A holographic setup for grating fabrication. (a) Exposure of photoresist by contradirectional laser beam derived from a common source. (b) Photoresist mask after development.

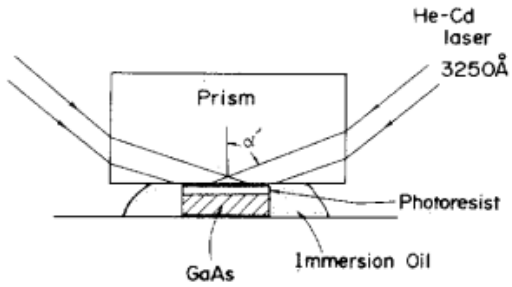

Fig. 9. A photographic exposure method for short period [4].

milling yield is a function of the beam energy, mass numbers of incident atoms and target atoms, etc. Typical milling rates are given in [15] and [16].

Surface corrugations produced in GaAs are shown in Figs. 10 and 11. In Fig. 10, a $0.41-\mu \mathrm{m}$ pattern was etched to a depth of $0.12 \mu \mathrm{m}$ into the surface of GaAs [8]. Fig. 11 shows a grating with a period of $0.12 \mu \mathrm{m}$ in $\mathrm{GaAs}$, where the depth was $\sim 0.05 \mu \mathrm{m}[14]$.

Substrate surfaces can also be corrugated by chemical etching. In semiconductors, for example, in $\mathrm{Si}$ and $\mathrm{GaAs}$, selective etching is a chemical technique which can be utilized to make well-defined grooves [17]-[19]. The usefulness of this method originates from the preferential etching habit of such single crystals. Fig. 12 shows the basic groove profiles that can be obtained when the (100) surface of GaAs is preferentially etched through a slotted photoresist mask. In both directions of the mask, the groove walls correspond to $A\{111\}$ crystallographic planes. These planes are selected because the etching rate for $A\{111\}$ planes is much slower than that for any other low-index planes. If the slotted mask is in the $\langle 01 \overline{1}\rangle$ direction, $V$-shaped grooves are easily produced [20]-[22]. Fig. 13 shows SEM photographs of the corrugated GaAs surface made by the preferential etching technique, with grating lines aligned along the $[01 \overline{1}]$ direction, and having a profile as shown in Fig. 12.

\section{REFleCtion Filters}

A corrugated section of a dielectric waveguide acts as a reflection filter [7] for frequencies near the forbidden gaps of the structure where $\Lambda \cong l \lambda_{g} / 2$, since only at these frequencies do the reflections from the corrugations build up in phase. This can be demonstrated if we plot the power reflection 


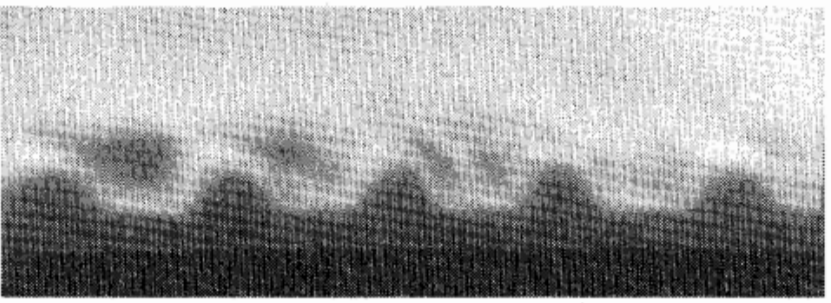

Fig. 10. An $0.411-\mu \mathrm{m}$ grating ion-etched into $\mathrm{GaAs}$ through a photoresist mask.

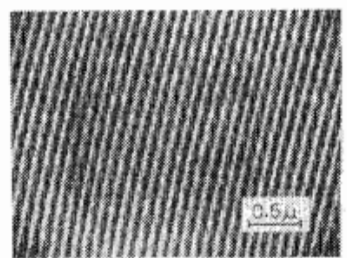

Fig. 11. An 0.12- $\mu$ m-period grating in GaAs.

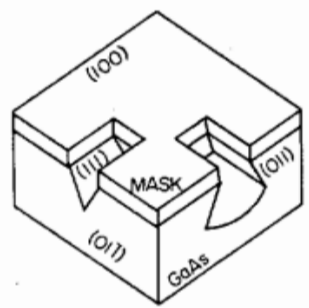

Fig. 12. Basic groove types observed when $\{100\}$ faces of GaAs are selectively etched. The $(01 \overline{1})$ and $(011)$ faces are cleaved planes [20].

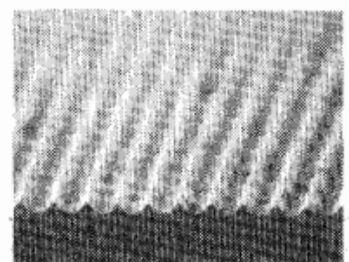

Fig. 13. A GaAs grating made by chemical etching. The grating period is $3470 \AA[21]$.

$|A(0) / B(0)|^{2}$ obtained from the first of (62) as a function of $\Delta \beta$ (in practice $\Delta \beta \equiv \beta-\pi l / \Lambda$ can be varied by varying the frequency). The result is shown in Fig. 14. Note that, according to (62), the maximum reflection coefficient obtains at $\Delta \beta=0$, i.e., the center of the forbidden "gap" and is

$$
R_{0}=\tanh ^{2}(|\kappa| L)
$$

and can thus approach unity for $|\kappa| L>>1$.

Bragg reflection filters were fabricated using sputtered glass waveguides [23]. The surface corrugation was fabricated by the holographic exposure technique and ion milling as described in the previous section. Fig. 15 is the illustration of a corrugated filter in a thin-film glass waveguide. The solid line denotes the experimental result, and the dashed line is the calculated reflectivity. The agreement between measured and calculated values is excellent in this example. Filters with $3-\mathrm{dB}$ bandwidth of less than $2 \AA$ and reflectivities greater than 75 percent were achieved in this structure.

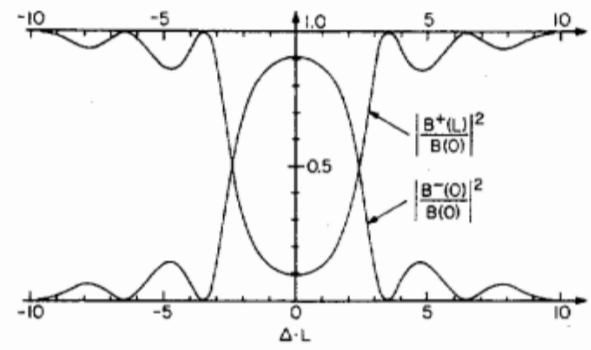

Fig. 14. The transmission and reflection coefficients of a corrugated section of a waveguide of length $L$ as a function of the detuning $\Delta \beta L$ $\simeq\left[\left(\omega-\omega_{0}\right) L n_{\mathrm{eff}} / c\right](\kappa L=1.84)$.

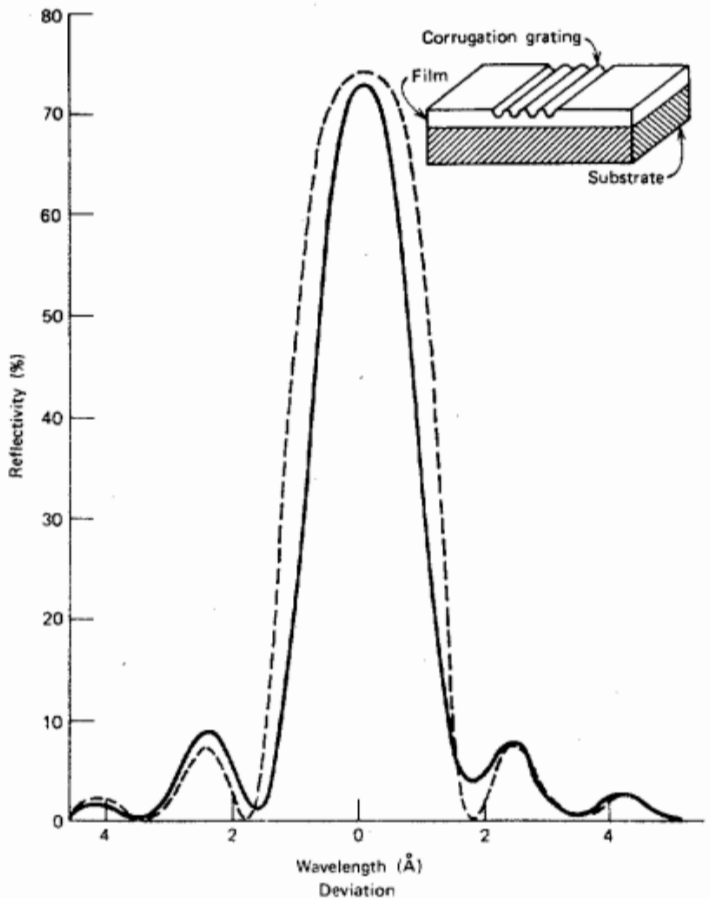

Fig. 15. Illustration of corrugation filter in a thin-film waveguide, plot (solid line) of reflectivity of filter versus wavelength deviation from the Bragg condition, and calculated response of filter (dotted line) $|A(0) / B(0)|^{2}$ using (62) (after [23]).

\section{Distributed Feedback LASERS}

Lasers in which the feedback is provided by a periodic perturbation rather than by reflectors are known as distributed feedback (DFB) lasers [24]. In what follows we will show how this form of laser oscillation can be treated within the framework of our theory and describe some types of DFB lasers.

\section{Threshold Condition}

If a corrugated guiding medium possesses gain, the coupled mode equations (61) are replaced by

$$
\begin{aligned}
& \frac{d A}{d z}=\kappa B^{-i 2(\Delta \beta) z}-g A, \\
& \frac{d B}{d z}=\kappa^{*} A \exp i 2(\Delta \beta) z+g B,
\end{aligned}
$$

where $g$ is the exponential gain constant of the unperturbed medium. In terms of mode amplitudes $\widetilde{A}(z)$ and $\widetilde{B}(z)$ defined 
by

$$
\begin{aligned}
& \tilde{A}(z)=A(z) e^{+g z}, \\
& \tilde{B}(z)=B(z) e^{-g z} ;
\end{aligned}
$$

equations (68) become

$$
\begin{aligned}
& \frac{d \tilde{A}}{d z}=\kappa \tilde{B} \exp [-i 2(\Delta \beta+i g) z], \\
& \frac{d \tilde{B}}{d z}=\kappa * \tilde{A} \exp [i 2(\Delta \beta+i g) z] .
\end{aligned}
$$

Equations (69) become identical to (61) if we replace $\Delta \beta \rightarrow$ $\Delta \beta+i g$. With this substitution, we can then use (62) to obtain the solutions for the complex field component $E_{y}(z)=\widetilde{B}(z)$ $\exp [(-i \beta+g) z]+\tilde{A}(z) \exp [(i \beta-g) z]$ in the periodic section of length $L$. Assuming an input incident field of $B(0)$ at $z=0$, as shown in Fig. 16, the solutions of (69) for the forward wave $E_{i} \equiv \tilde{B} \exp [(-i \beta+g) z]$ and the backward wave $E_{r} \equiv \tilde{A} \exp$ $[(i \beta-g) z]$ are

$$
E_{i}(z)=
$$

$B(0) \frac{e^{-i \beta_{0} z}\{(g-i \Delta \beta) \sinh [\gamma(L-z)]-\gamma \cosh [\gamma(L-z)]\}}{(g-i \Delta \beta) \sinh (\gamma L)-\gamma \cosh (\gamma L)}$

$$
E_{r}(z)=B(0) \frac{e^{i \beta_{0} z} \sinh [\gamma(L-z)]}{(g-i \Delta \beta) \sinh (\gamma L)-\gamma \cosh (\gamma L)}
$$

where

$$
\gamma^{2}=|\kappa|^{2}+(g-i \Delta \beta)^{2} .
$$

The fact that $\gamma$ is now complex makes for a qualitative difference between the behavior of the passive periodic waveguide (62) and the periodic waveguide with gain (70). In (70), the amplitude ratios $E_{i}(L) / B(0)$ and $E_{r}(0) / B(0)$ become infinite when the condition

$$
(g-i \Delta \beta) \sinh (\gamma L)=\cosh (\gamma L)
$$

is satisfied. This corresponds to finite output fields $E_{r}(0)$ and $E_{i}(L)$ with no input $(B(0)=0)$. The device then acts as an oscillator, and (72) is the threshold condition for DFB lasers [26], [28]. It can be written as

$$
\frac{\gamma-(g-i \Delta \beta)}{\gamma+(g+i \Delta \beta)} e^{2 \gamma L}=-1 \text {. }
$$

The reflection gain $\left|E_{r}(0) / E_{i}(0)\right|^{2}$ and the transmission gain $\left|E_{i}(L) / E_{i}(0)\right|^{2}$ are plotted in Figs. 17 and 18 , respectively. Each plot contains four infinite gain singularities at which the oscillation condition (73) is satisfied [28]. These are four of the longitudinal laser modes. It should be noted that the longitudinal mode closest to the Bragg frequency $\omega_{0}$ has the lowest threshold gain. This fact means that DFB lasers have longitudinal mode selectivity.

In general, one has to resort to a numerical solution to obtain the threshold values of $\Delta \beta$ and $g$ for oscillation. In some limiting cases, however, we can obtain approximate solutions. In the high-gain $g$ case with index modulation, equation (73) becomes

$$
\frac{4(g-i \Delta \beta)^{2}}{n^{2}} e^{2} \gamma L=-1 .
$$

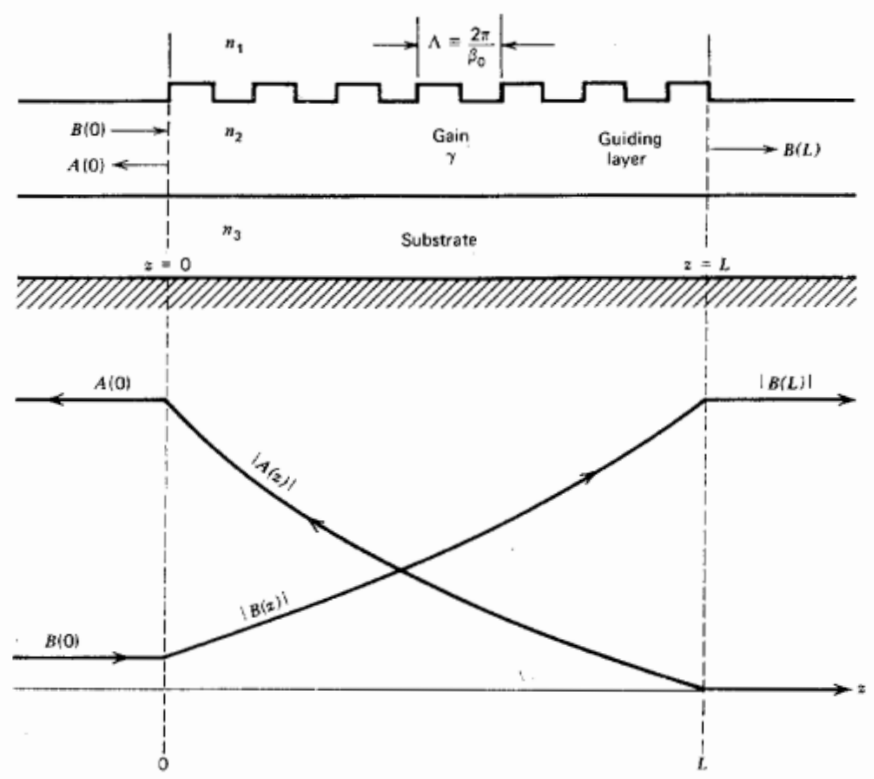

Fig. 16. The incident and reflected fields inside an amplifying periodic waveguide near the Bragg condition $\beta \approx \pi / \Lambda$.

Equating the phases on both sides of (74) results in

$$
\begin{aligned}
(\Delta \beta) L & \simeq\left(n+\frac{1}{2}\right) \pi \\
n & =0, \pm 1, \pm 2, \cdots,
\end{aligned}
$$

where $n$ denotes the longitudinal mode. Since $\Delta \beta \equiv \beta-\beta_{0} \simeq$ $\left(\omega-\omega_{0}\right) n_{\text {eff }} / c$,

$$
\omega_{n}=\omega_{0}-\left(n+\frac{1}{2}\right) \frac{\pi c}{n_{\mathrm{eff}} L} .
$$

We note that no oscillation takes place at the Bragg frequency $\omega_{0}$. The mode frequency spacing is

$$
\omega_{n}-\omega_{n-1}=\frac{\pi c}{n_{\mathrm{eff}} L}
$$

and is approximately the same as in a Fabry-Perot resonator. The threshold gain value is obtained from the amplitude equality in (74),

$$
\frac{e^{2 g} n^{L}}{g_{n}^{2}+(\Delta \beta)_{n}^{2}}=\frac{4}{\kappa^{2}}
$$

indicating an increase in threshold with increasing mode number $n$.

In the limit of low gain $g<<\kappa$, we have from (73)

$$
\Delta \beta \approx \kappa
$$

and

$$
g L \approx\left(\frac{\pi}{\kappa L}\right)^{2} .
$$

Numerical solutions of (72) were given in many references [24]-[29].

\section{DFB Dye Lasers}

Laser action was first demonstrated in dye lasers [30]-[38] . Kogelnik et al. [30] demonstrated a DFB dye laser in a gelatin 


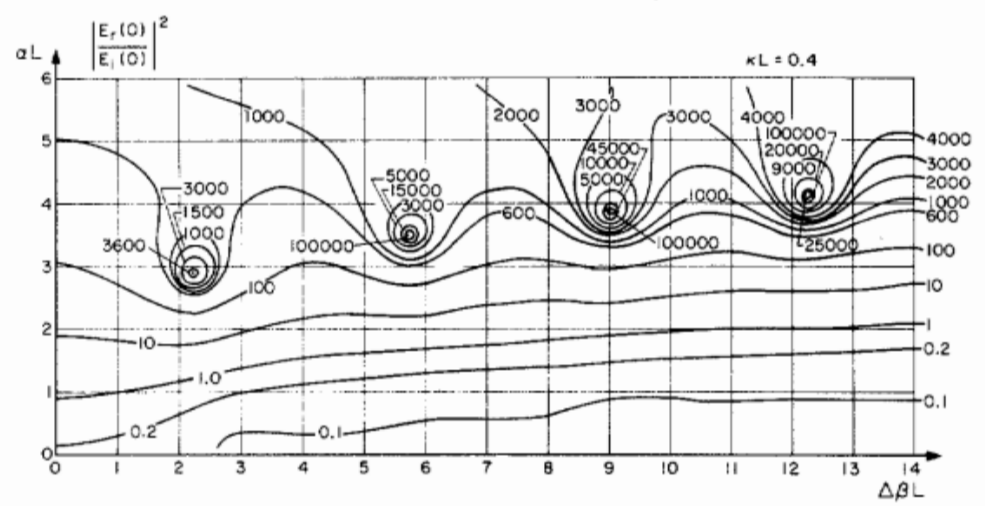

Fig. 17. Reflection gain contours in the $\Delta \beta L-\gamma L$ plane for a periodic waveguide with $\kappa L=0.4$.

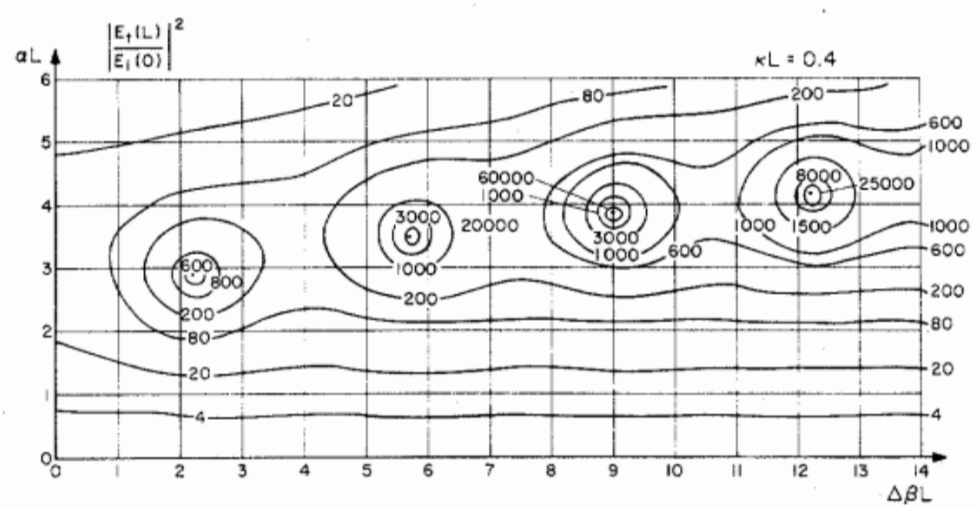

Fig. 18. Transmission gain contours in the $\Delta \beta L-\gamma L$ plane for a periodic waveguide with $\kappa L=0.4$.

film as shown in Fig. 19. The laser was $10 \mathrm{~mm}$ long and about $0.1 \mathrm{~mm}$ wide. The gelatin was dichromated and exposed to the interference pattern produced by two coherent beams from an $\mathrm{He}-\mathrm{Cd}$ laser. The period was $\sim 0.3 \mu \mathrm{m}$. The development of the gelatin resulted in a spatial modulation of the refractive index. The developed gelatin was soaked in a solution of rhodamine $6 \mathrm{G}$. The dye was pumped by a nitrogen laser beam, and laser oscillation was observed at a wavelength of $\sim 0.63 \mu \mathrm{m}$, with a linewidth of less than $0.5 \AA$.

The frequency selectivity in DFB lasers was clearly demonstrated by changing the period of modulation as shown in Fig. 20 [31]. In this experiment, the $0.347-\mu \mathrm{m}$-wavelength beam was split with a beam splitter into two nearly equal parts, and recombined at the rhodamine $6 \mathrm{G}$ dye cell, resulting in the periodic modulation of gain (and refractive index) in the dye. The lasing wavelength $\lambda$ was found to satisfy the Bragg condition

$$
\lambda \cong n_{\text {eff }} \lambda_{p} / \sin \theta
$$

where $\lambda_{p}$ is the wavelength of the pumping light and $\theta$ is the incident angle. The laser showed single longitudinal mode operation with a linewidth of $\sim 0.01 \AA$ at low excitation levels.

The threshold gain of DFB dye lasers was examined by using corrugated waveguide structures [9], [38]. The threshold gain $g$ is in agreement with (80)

$$
g L \approx(\pi / \kappa L)^{2} .
$$

The pumping intensity $I_{p}$ and the resulting gain constant $g$ are related by

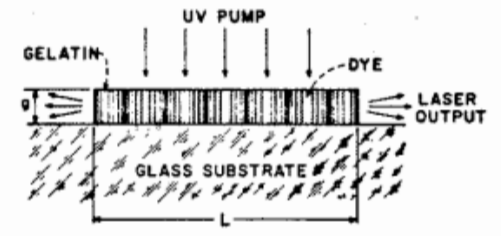

Fig. 19. Cross section of a DFB laser consisting of dyed gelatin on a glass substrate [35].

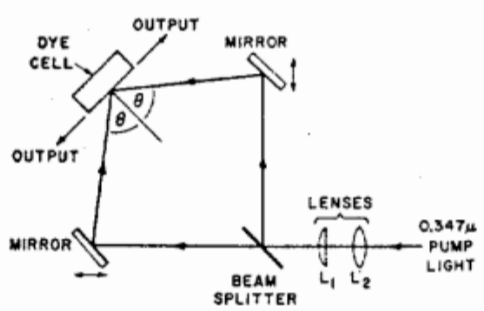

Fig. 20. Schematic diagram of tunable DFB dye laser [36].

$$
g=G I_{p},
$$

where $G$ is the gain coefficient of the medium. The threshold pumping $I_{p t}$ is thus given by

$$
g_{\text {tot }}=\alpha_{\text {loss }}+(\pi / \kappa)^{2} / L^{3}=G I_{p t} \text {, }
$$

where $\alpha_{\text {loss }}$ is the loss constant of the waveguide. The corrugated dye laser was pumped at right angles to the plane of the film by a pulsed $N_{2}$ laser. The threshold pump intensity $I_{p t}$ was measured as a function of the pumping length $L$ and shown in Fig. 21. The intercept on the ordinate $(L \rightarrow \infty)$ gives 


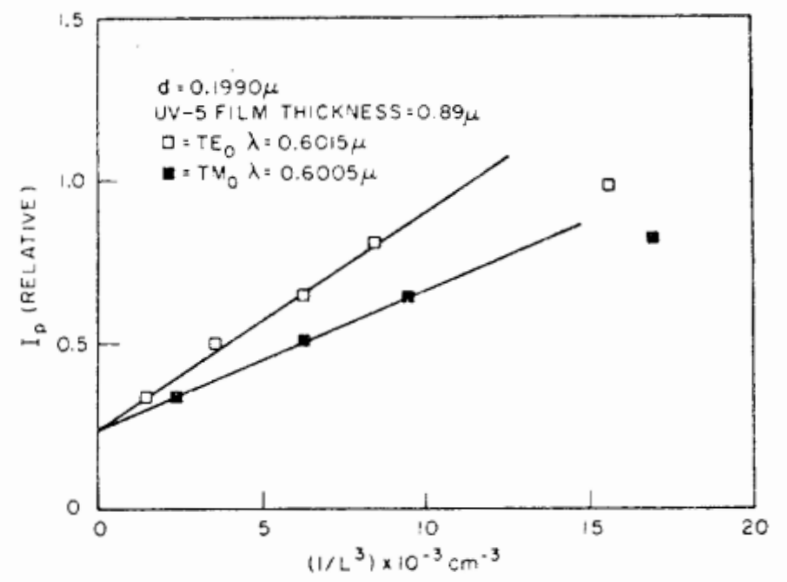

Fig. 21. Threshold pump intensity as a function of pumped region. The straight lines are the expected dependence (32) [11].

the intrinsic loss of the guided mode $\alpha_{\text {loss }}$, while the slope of the curve depends on the coupling constant $\kappa$. The data are in reasonable agreement with the theoretical dependence as given by (83). The gain coefficient $G$ in (83) was determined from the measurements of amplified spontaneous emission [41], and $G \approx 25-55 \mathrm{~dB} / \mathrm{cm}$. Using these values and the slopes in Fig. 21, the coupling constant $\kappa$ was estimated to range from 12 to $780 \mathrm{~cm}^{-1}$, which agreed well with the theoretical values.

\section{DFB Semiconductor Lasers}

The most important application of the DFB principle is probably in the case of semiconductor injection lasers. This is due to the important role these lasers play in optical communication and to the fact that the use of DFB offers the first viable solution to controlling selectively their output spectrum. In addition the corrugation techniques developed for these lasers are consistent with semiconductor planar processing and with integrated optical circuits.

The first experiments were performed on GaAs surfacecorrugated samples pumped optically [39]-[41] . A schematic structure of a DFB GaAs surface laser is shown in Fig. 22. Surface corrugations with a period of $0.115 \mu \mathrm{m}$ (first order) or $0.345 \mu \mathrm{m}$ (third order) were fabricated by photographic photolithography and ion milling, and the GaAs surface was pumped by ruby laser beams [39] and dye laser beams [40], [41]. Lasing occurred with a threshold pumping intensity of $10^{4} \mathrm{~W} / \mathrm{cm}^{2}$ at $77 \mathrm{~K}$. The threshold power gain $2 g_{\text {tot }}$ was estimated to be $100 \mathrm{~cm}^{-1}$ as shown in Fig. 23 [40]. The threshold gain was obtained assuming a linear dependence of the exponential gain constant on the pumping density as in (83). The constant $G$ was estimated to be $\sim 5 \mathrm{~cm} / \mathrm{kW}$ from the gain measurement. In Fig. 23, the solid line denotes the theoretical value calculated from (72) for $\kappa=1.93 \mathrm{~cm}^{-1}$ and $\alpha_{\text {loss }}=15 \mathrm{~cm}^{-1}$ which were obtained from other experiments.

Injection lasers with corrugated interface were made in single heterostructure [42], [43] homostructure, and double heterostructure [44]-[46], and operated at low temperatures. In the single heterostructure and double heterostructure lasers, an additional GaAlAs layer was regrown on a corrugated surface of a GaAs layer. The emission spectra of a typical injection

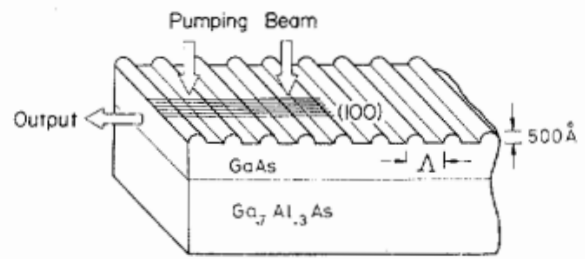

Fig. 22. Schematic structure of a GaAs DFB laser. $\Lambda \sim 0.35 \mu \mathrm{m} \mathrm{[45],}$ [46] and $\sim 0.11 \mu \mathrm{m} \mathrm{[4]}$.

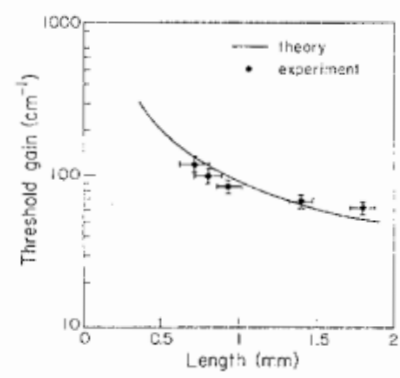

Fig. 23. Threshold gain of a DFB GaAs laser $2 g_{\text {tot }}$ as a function of the excitation length $L$ [46].

laser are shown in Fig. 24. The period of the corrugation is $3470 \AA$, the length of the active region is $1050 \mu \mathrm{m}$, and the threshold current density is $750 \mathrm{~A} / \mathrm{cm}^{2}$ at $80 \mathrm{~K}$. Just above threshold $(390 \mathrm{~mA})$, a narrow peak of stimulated emission is shown at $8225 \AA$. The linewidth of the stimulated emission was $\approx 0.5 \AA$. Diodes lased usually in a single longitudinal mode up to twice the threshold pumping. Fig. 25 shows the spectrum of a typical double heterostructure laser as a function of temperature. In this experiment, the current was chosen to be about $\sim 1.05$ times the threshold. In Fig. 25, the peak wavelength of spontaneous emission shifts more rapidly than the peak of the stimulated emission. The temperature dependence of the lasing wavelength is given, according to the Bragg condition, by

$$
\frac{d \lambda}{d T}=\frac{\lambda}{\bar{n}_{\text {eff }}} \frac{\partial n_{\text {eff }}}{\partial T},
$$

where

$$
\bar{n}_{\text {eff }}=n_{\text {eff }}-\frac{\partial n_{\text {eff }}}{\partial \lambda} \lambda .
$$

With $\bar{n}_{\text {eff }} \simeq 4.5, \partial n_{\text {eff }} / \partial T \simeq 3 \times 10^{-4} /$ degrees [47], we have $d \lambda / d T \simeq 0.6 \AA /$ degrees, which agrees well with the experimental results shown in Fig. 25. The peak wavelength of the spontaneous emission, on the other hand, shifts (see Fig. 27) at a rate of $\sim 2 \AA /$ degrees at $\sim 100 \mathrm{~K}$ [21] which is essentially the shift of the energy gap (expressed in equivalent wavelength) as a function of temperature.

The problem inherent in the above DFB semiconductor diode lasers was the steep increase of threshold current density at higher temperatures. It was found that the fabrication of a grating on the active layer caused interface recombination centers which increased the threshold current density substantially at higher temperatures [48]. This problem has been overcome by adopting separate confinement heterostructures $(\mathrm{SCH})$ [49]-[51]. An example of SCH corrugated 


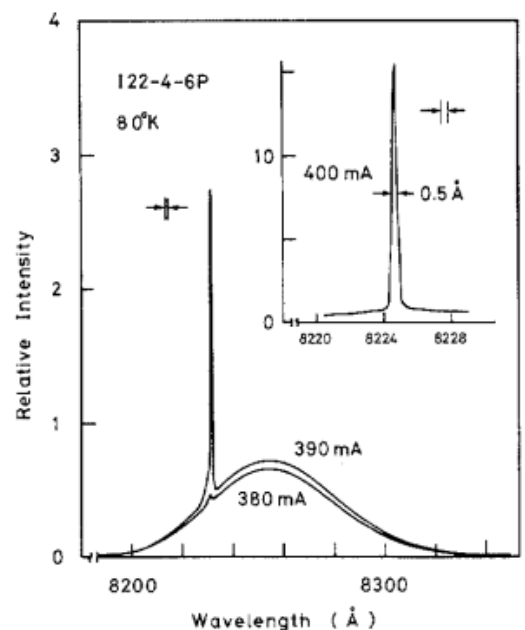

Fig. 24. The emission spectra of a typical DFB GaAs injection laser. The period and the depth of the corrugation are $3470 \AA$ and $1800 \AA$, respectively. The threshold current density is $750 \mathrm{~A} / \mathrm{cm}^{2}$ at $80 \mathrm{~K}$ [21].

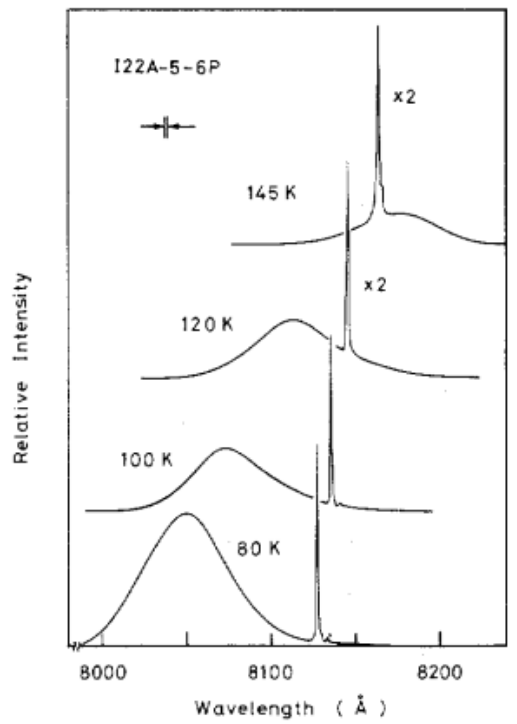

Fig. 25. Temperature dependence of the emission spectrum of a DFB GaAs injection laser. The current is chosen to be $\sim 1.05$ times the threshold for each temperature [21].

waveguides is shown in Fig. 26. In this structure the injected electrons are confined to the $\mathrm{p}-\mathrm{GaAs}$ active layer, while the mode profile extends to the $\mathrm{p}-\mathrm{Ga}_{1-y} \mathrm{Al}_{y}$ As layer, $(y \sim 0.17)$ and the $\mathrm{p}-\mathrm{Ga}_{1-z} \mathrm{Al}_{z}$ As layer $(z \sim 0.07)$ grown successively on the active layer. Since the active layer was separated from the corrugated interface, the threshold current density has been low enough to operate the diode at room temperature. A similar structure was also reported by Casey et al. [49], where the grating was fabricated on the $\mathrm{p}-\mathrm{Ga}_{1-y} \mathrm{Al}_{y} \mathrm{As}$ layer and $\mathrm{p}-\mathrm{Ga}_{1-x} \mathrm{As}_{x}$ As layer $(x \sim 0.3)$ was deposited on it by molecular beam epitaxy.

The threshold current density and lasing wavelength of an SCH DFB laser are plotted by open circles in Fig. 27 [51]. In this laser, three different modes were observed. The lowest threshold current density was $3 \mathrm{kA} / \mathrm{cm}^{2}$ at $320 \mathrm{~K}$. The diode was also operated under $\mathrm{CW}$ bias. The dots in Fig. 27 are the results obtained in a cleaved laser made from the same wafer.

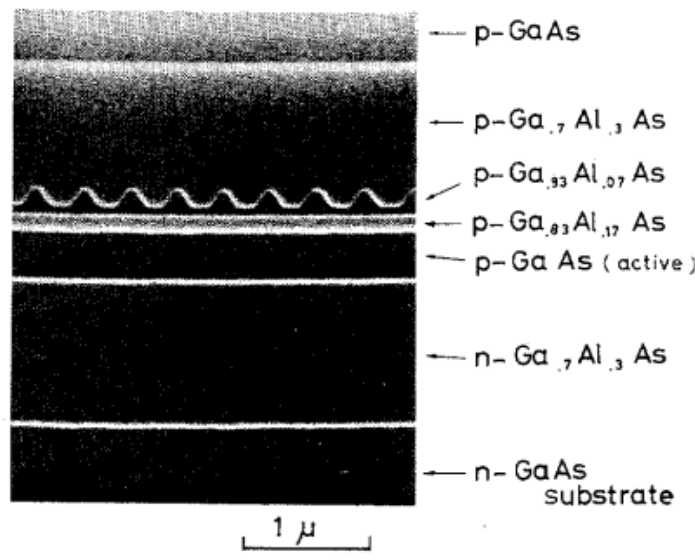

Fig. 26. SEM photograph of the cross section of a separate confinement heterostructure DFB GaAs-GaAlAs laser [59].

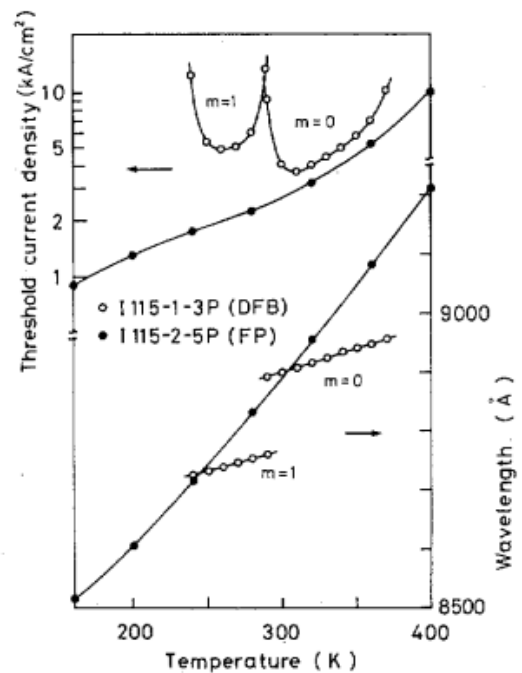

Fig. 27. Threshold current density and lasing wavelength of a DFB $\mathrm{SCH}$ structure $\mathrm{GaAs}$ laser (open circles) and those of a cleaved laser (dots) [59].

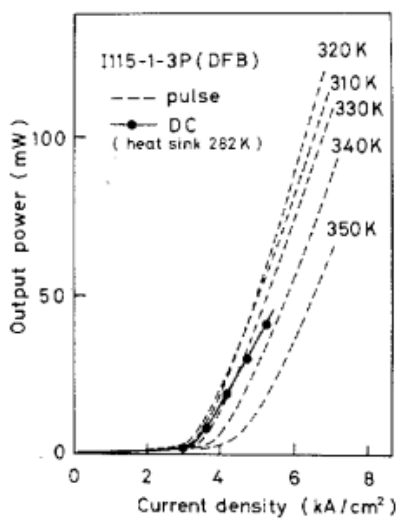

Fig. 28. Output power of a DFB GaAs injection laser as a function of applied current density. The stripe width was $50 \mu \mathrm{m}$ [59].

The output power of an SCH DFB laser is shown in Fig. 28 as a function of applied current. An output power as high as $\sim 60 \mathrm{~mW}$ was obtained under $\mathrm{CW}$ operation. The differential quantum efficiency $n_{\text {ext }}$ was $\sim 7$ percent under pulse bias, and $\sim 5$ percent under dc bias from one facet. 


\section{Grating Coupled Lasers}

In DFB lasers in which a high grating order $(l=2,3)$ is used to obtain feedback, the low diffraction orders of the same grating lead to coupling to radiation modes and thus to output beams coupled with a low-angle divergence. Grating couplers, which will be considered in the next section, were also incorporated into conventional cleaved-ends semiconductor lasers to obtain low-divergence output beams [52], [53]. In these lasers, the optical feedback was provided by the cleaved ends and light traveling in the waveguide was diffracted by the corrugated interface. The exit angle in air is given by (35) (replacing $\theta$ by $\pi / 2-\phi$, putting $n_{1}=1$ and $\beta \equiv 2 \pi / \lambda \mathrm{n}_{\mathrm{eff}}$ )

$$
\sin \phi=l \lambda / \Lambda-n_{\mathrm{eff}}
$$

where $\phi$ is the angle of the wave normal measured from the normal to the surface, and $l$ is the diffraction order integer.

Consider a DFB laser with a period of $\Lambda=l \lambda / n_{\text {eff }}$, where optical feedback is provided by the Bragg scattering of order $2 l$. Bragg scattering of order $l$ then results in an output beam emanating at a right angle to the junction plane. In a DFB laser with a long active region, the angular divergence of the output beam $\Delta \phi$ is obtained by differentiating (86)

$$
\Delta \phi=\left(\frac{p}{\Lambda}-\frac{d n_{\mathrm{eff}}}{d \lambda}\right) \Delta \lambda
$$

where $\Delta \lambda$ is the spectral width of the radiation. For a nearmonochromatic laser, $\Delta \phi$ is determined by the diffraction limit of the grating aperture. An angular scan of the far-field pattern of radiation from a GaAs-GaAlAs single heterostructure DFB laser is shown in Fig. 29. Corrugations with a period of $4693 \AA$ corresponding to a fourth-order DFB grating were fabricated in the laser. The output beams by the first and third-order diffraction were not observed due to the internal total reflection. The divergence of the beam (which is coupled in second order $l=2$ ) in the direction along the laser length was measured to be $\sim 0.35^{\circ}$.

\section{Distributed Bragg Reflector Lasers}

\section{Threshold Condition}

An alternative to the DFB laser is one where the Bragg coupling between the forward and backward waves is achieved in two corrugated waveguide sections outside the active region, as shown in Fig. 30. The corrugated sections with lengths of $L_{1}$ and $L_{3}$ can act as high-reflectance "mirrors" for waveguide modes at frequencies within the "forbidden gap" as was discussed in Section V. Such DBR lasers possess the main advantages of the DFB lasers, i.e., frequency and mode selectivity, while avoiding the problem of degradation of the recombination efficiency by the corrugated interface.

Theoretical analyses of DBR lasers were given by a number of authors [57]-[61]. Formally, the periodically corrugated sections of the waveguide can be replaced by fictious reflectors with complex reflectances $r_{1,2}(\omega) \exp \left[-i \phi_{1,2}(\omega)\right]$. The complex reflectance of each reflector is given, from (62), by

$$
r(\omega) \exp [-i \phi(\omega)]=\frac{i \kappa \sinh (\gamma L)}{(\alpha+i \Delta \beta) \sinh (\gamma L)+\gamma \cosh (\gamma L)}
$$

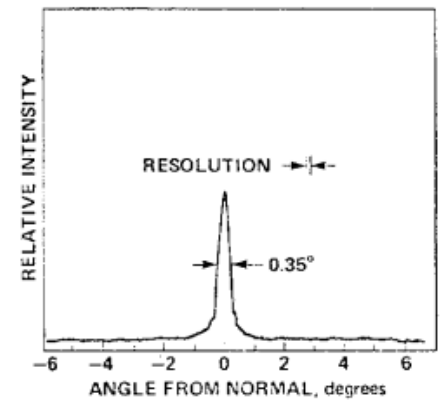

Fig. 29. Angular scan of the far-field radiation pattern in the direction perpendicular to the corrugation grooves. Angular resolution is $\sim 0.1^{\circ}[63]$.

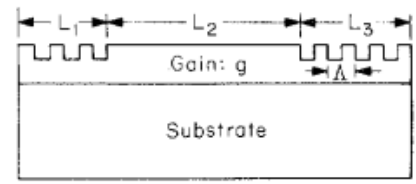

(a)

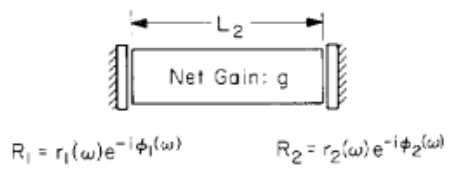

(b)

Fig. 30. (a) Schematic diagram of a DBR laser. (b) Equivalent cavity of a DBR laser [59].

where $\alpha$ is the loss constant of the corrugated section, $\gamma^{2}=$ $\kappa^{2}+(\alpha+i \Delta \beta)^{2}$, and $L$ stands for the length of each corrugated section. The lasing condition is then obtained by setting the round trip gain equal to unity, i.e.,

$$
\begin{gathered}
r_{1}(\omega) r_{2}(\omega) \exp -i\left[\phi_{1}(\omega)+\phi_{2}(\omega)\right] \\
\cdot \exp 2\left[(g(\omega)-i \beta(\omega)] L_{2}=1,\right.
\end{gathered}
$$

where $L_{2}$ is the length of the active region. Using (88), (89) becomes

$$
\begin{aligned}
\kappa^{2} \exp \left[2(g-i \beta) L_{2}\right]= & {\left[(\alpha+i \Delta \beta)+\gamma \operatorname{coth}\left(\gamma L_{1}\right)\right] } \\
& \cdot\left[(\alpha+i \Delta \beta)+\gamma \cot \left(\gamma L_{3}\right)\right] .
\end{aligned}
$$

This is the eigenvalue equation of a DBR laser.

Equation (90) can be solved graphically [68]. From (89), one gets

$$
\begin{aligned}
g & =\frac{1}{2 L_{2}} \ln \frac{1}{r_{1} r_{2}}, \\
\Phi & \equiv \phi_{1}+\phi_{2}+2 \Delta \beta L_{2}+2 \beta_{0} L_{2}=2 n \pi, \\
n & =0, \pm 1, \pm 2, \cdots .
\end{aligned}
$$

In a given DBR structure, we use (88) to obtain $r_{1,2}$ and $\phi_{1,2}$ as a function of $\Delta \beta$. Then the round trip phase delay $\Phi$ is plotted as a function of $\Delta \beta$. The intersections of this curve with horizontal lines $\Phi=2 n \pi$ determine the oscillation frequencies $\Delta \beta_{n}$. The corresponding $r_{1}\left(\Delta \beta_{n}\right)$ and $r_{2}\left(\Delta \beta_{n}\right)$ are then used in (91) to find the threshold gain $g_{n}$. This procedure is illustrated in Fig. 31 for a case of $L_{1}=L_{3}=L, L_{2}=2 L$, $\kappa L=3.0, \alpha=0$, and $\beta_{0} L=2 \pi$. 
(a)

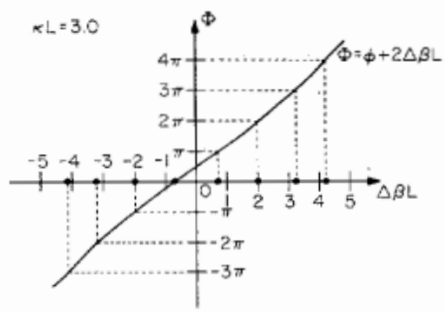

(b)

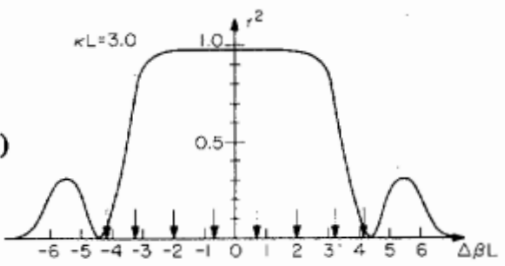

Fig. 31. Graphical method of determining the oscillation condition of a DBR laser. (a) Plots of $\Phi=\Phi+2 \Delta \beta L$ and $=n \pi$. The intersections give the frequencies of oscillation. (b) The thresholds are determined using the values of reflectivity at frequencies found in (a) [59].

\section{Distributed Bragg Reflector Semiconductor Lasers}

DBR lasers have been fabricated in a GaAs-GaAlAs crystal. They have been pumped optically [59] and by current injection [61]-[63]. A schematic diagram of the layer structure of this laser is shown in Fig. 32. The spectrum of a roomtemperature DBR laser is shown in Fig. 33. The basic tendency of the laser to oscillate in a single mode is evident. This class of lasers has been developed only recently and experimental results are still sparse.

\section{Grating Couplers}

Diffraction gratings built directly into a dielectric waveguide as in Fig. 34 can be used as input or output couplers from the waveguide (confined) modes to free space, or substrate, propagating modes. This application was first illustrated by Dakss et al. [6] . It was also discussed in Section VII.

The qualitative explanation of the operation of a grating coupler was given in Section III and is illustrated in Fig. 4(b), A number of theoretical analyses exist for calculating the coupling efficiency of such gratings. These analyses use somewhat different approaches [64]-[70]. The theoretical approach closest to the spirit of this paper is that using the formalism of Floquet modes best exemplified by [69], [70] .

In this formalism one solves the wave equation

$$
\frac{\partial^{2} E y}{\partial x^{2}}+\frac{\partial^{2} E y}{\partial z^{2}}+k^{2} n^{2}(x, z) E_{y}=0,
$$

where $k=\omega / c$ and the $n(x, z)$ is the spatial distribution of the index of refraction including the effect of the grating. Since $n^{2}(x, z)$ is a periodic function of $z$, the period $\Lambda$ being that of the grating, the solution must be given, according to the Floquet theorem, by an infinite sum of partial waves (often referred to as spatial harmonics)

$$
E_{y}=\sum_{m=-\infty}^{\infty} \varepsilon_{m}(x) \exp \left[-i\left(\beta_{0}+m \frac{2 \pi}{\Lambda}\right) z\right] .
$$

If the grating height is shrunk gradually to zero, all the $\varepsilon_{m}$

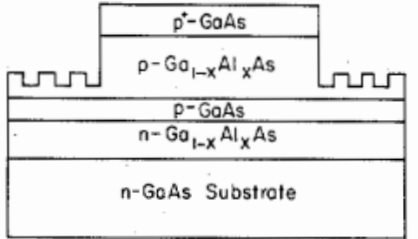

(a)

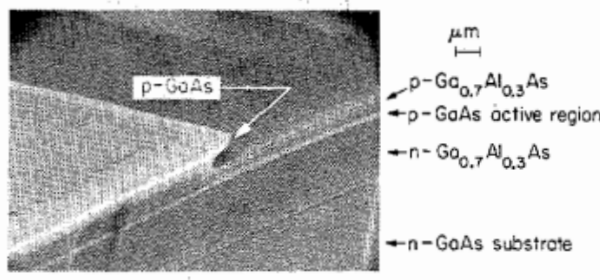

(b)

Fig. 32. (a) Schematic diagram of GaAs-GaAlAs injection laser with Bragg reflectors. (b) SEM of a double heterostructure GaAs-GaAlAs injection laser with distributed Bragg reflectors [63].
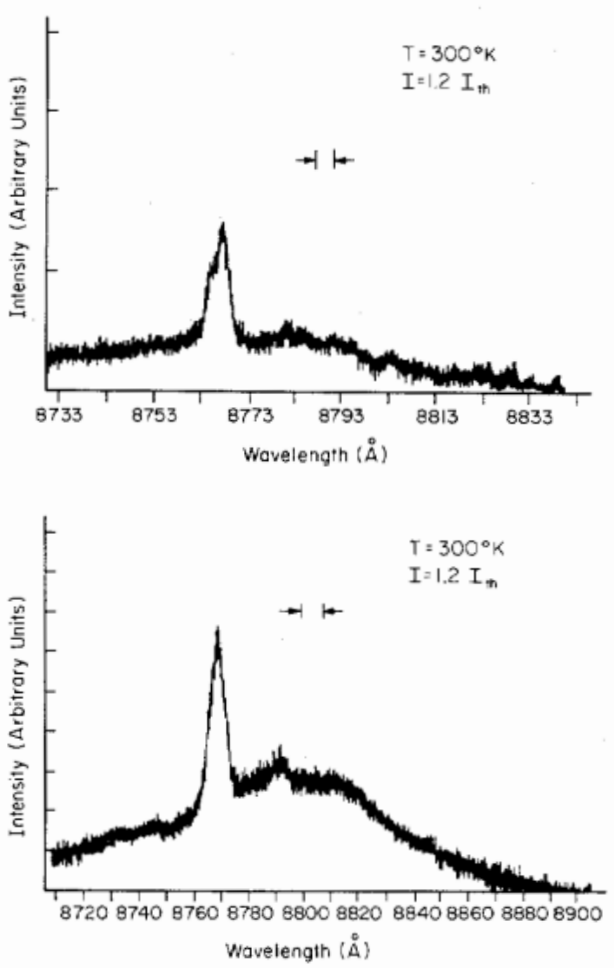

Fig. 33. Typical single spectra of the laser shown in the previous figure.

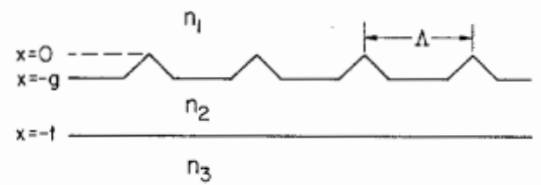

Fig. 34. A schematic diagram of a waveguide grating coupler.

functions become zero except $\mathscr{E}_{0}(x)$ which approaches the mode solution of the uniform waveguide. The solution of the problem thus consists of determining $\beta_{0}$ and $\AA_{m}(x)$. Let us consider a given mode, say $m$, in region 1 of Fig. 34. The power flow in this mode in the transverse $(+x)$ direction (in 
watts per square meter) is

$$
P_{m}=\frac{1}{2} \operatorname{Re}\left[E_{y m} H_{z m}^{*}\right] \text {. }
$$

Since region 1 is homogeneous, the nature of the wave solution of mode $m$ is that of a plane wave of the form

$$
E_{y m}(x, z)=\mathscr{\varepsilon}_{m} \exp \left[-i\left(k_{x m} x+\beta_{m} z\right)\right]
$$

where

$$
\begin{aligned}
\beta_{m} & =\beta_{0}+\frac{2 \pi}{\Lambda} m, \\
k_{x m} & =\sqrt{\left(\frac{\omega}{c}\right)^{2} n_{1}^{2}-\left(\beta_{0}+m \frac{2 \pi}{\Lambda}\right)^{2}} ;
\end{aligned}
$$

so that as required by (93)

$$
k_{x m}^{2}+\beta_{m}^{2}=\left(\frac{\omega}{c}\right) n_{1}^{2} .
$$

Using (96) in (95) as well as the relation

$$
H_{z}=\frac{i}{\omega \mu_{0}} \frac{\partial E_{y}}{\partial x}
$$

which holds for TE modes in slab $(\partial / \partial y=0)$ waveguides, $(95)$ becomes

$$
\begin{aligned}
P_{m} & =\frac{1}{2 \omega \mu_{0}}\left\{\left|\mathscr{E}_{m}(0)\right|^{2} \operatorname{Re}\left(k_{x m}\right)\right\} \\
& =\frac{\left|\mathscr{E}_{m}(0)\right|^{2}}{2 \omega \mu_{0}} \operatorname{Re}\left[\sqrt{\frac{\omega^{2}}{c^{2}} n_{1}^{2}-\left(\beta_{0}+m \frac{2 \pi}{\Lambda}\right)^{2}}\right],
\end{aligned}
$$

where the power flow is calculated for convenience at $x=0^{+}$, i.e., just outside the corrugation region. A similar expression but with $\mathscr{E}_{m}(0)$ replaced by $\mathscr{E}_{m}(-t)$ will give the power flow per unit area into the substrate $n_{3}$ (see Fig. 34 for the relevant geometry).

Consider a case where the dominant spatial harmonic $(m=0)$ satisfies the condition

$$
\beta_{0}>\frac{\omega}{c} n_{1}
$$

According to (96) $k_{x 0}$ is imaginary and the harmonic $m=0$ is thus evanescent in region (1) $\left(P_{0}=0\right)$. Another harmonic $m \neq 0$, however, may exist for which $k_{x m}$ is real. (This, of course, will depend on the magnitude of the grating period $\Lambda$ ). This harmonic will according to (98) radiate into region I. The exponential attenuation constant $\alpha$ of a propagating mode after entering the grating region is thus given by

$$
\alpha=\sum_{m=-\infty}^{\infty} P_{m} /\left\{\left(\beta_{0} / 2 \omega \mu_{0}\right) \int_{-\infty}^{\infty}\left|\varepsilon_{0}(x)\right|^{2} d x\right\},
$$

which is the ratio of $\Sigma_{m} P_{m}$, the power radiated per unit waveguide length by all the high-order harmonics, to the power in the fundamental $(m=0)$ harmonic. Although the summation in (99) extends from $m=-\infty$ to $m=\infty$, the number of modes for which $k_{x m}$ is real (so that $P_{m} \neq 0$ ) is always finite and in practice it can be made small so that the summation in (99) may include only one or two terms.

The deviation of $\alpha$ thus requires a solution for the spatial harmonics profiles $\mathscr{E}_{m}(x)$. If we express the index of refraction of a corrugated waveguide as in (48) by a Fourier series

$$
n^{2}(x, z)=\sum_{q=-\infty}^{\infty} a_{q}(x) \exp i \frac{2 \pi q z}{\Lambda}
$$

and then substitute in (93) we obtain, using (94),

$$
\begin{aligned}
& \sum_{m}\left[\frac{\partial^{2} \varepsilon_{m}}{\partial x^{2}}-\beta_{m}^{2} \varepsilon_{m}(x)\right] \exp \left(-i \beta_{m} z\right) \\
& \quad=-\frac{\omega^{2}}{c^{2}} \sum_{q} \sum_{m^{\prime}} a_{q}(x) \varepsilon_{m} \cdot \exp \left[-i\left(\beta_{m^{\prime}}-\frac{2 \pi}{\Lambda} q\right) z\right]
\end{aligned}
$$

where

$$
\beta_{m}=\beta_{0}+m \frac{2 \pi}{\Lambda}
$$

Equating terms with the same $z$ dependence leads to

$$
\begin{aligned}
\frac{\partial^{2} \&_{m}}{\partial x^{2}} & +\frac{\omega^{2}}{c^{2}} a_{0}(x) \&_{m}-\beta_{m}^{2} \&_{m} \\
= & -\frac{\omega^{2}}{c^{2}} \sum_{q \neq 0} a_{q}(x) \&_{q+m}(x) .
\end{aligned}
$$

An iterative solution of (103) can yield excellent approximate solutions for $\varepsilon_{m}(x)$. Such procedures were described by a number of authors [64], [71], [60] and most recently by Streifer et al. [69], [70]. We first obtain the zeroth order solution by setting the right-hand side of (103) equal to zero and then solving for $\varepsilon_{0}(x)$. The function $\varepsilon_{0}(x)$ thus obtained is used next in (103) resulting in

$$
\begin{gathered}
\frac{\partial^{2} \varepsilon_{-1}}{\partial x^{2}}+\frac{\omega^{2}}{c^{2}} a_{0}(x) \varepsilon_{-1}-\left(\beta_{0}-\frac{2 \pi}{\Lambda}\right)^{2} \varepsilon_{-1} \\
=-\frac{\omega^{2}}{c^{2}} a_{1}(x) \varepsilon_{0}(x) .
\end{gathered}
$$

Equation (104) can be solved for $\mathscr{E}_{-1}(x)$ which is used in (99) to obtain the loss constant due to radiation into the $m=$ -1 order.

A result of such a calculation taken from [70] is shown in Fig. 35 for the case of a triangular corrugation. Note the symmetry of the curves about the value $\delta=0.5$ (the slope parameter $\delta=\Delta / \Lambda$ is defined in the figure). The curve shown is for propagation from left to right. For the opposite direction of propagation the loss $\alpha$ is obtained at a value of the shape parameter $1-\delta$. From the figure it follows that except for the case of a symmetric corrugation $(\delta=0.5)$, the power loss exercised by modes traveling in opposite directions is not equal and can differ by a large factor. This is analogous to the blazing effect in conventional spectroscopic gratings. This effect has been demonstrated by Aoyagi and Namba [72] . 


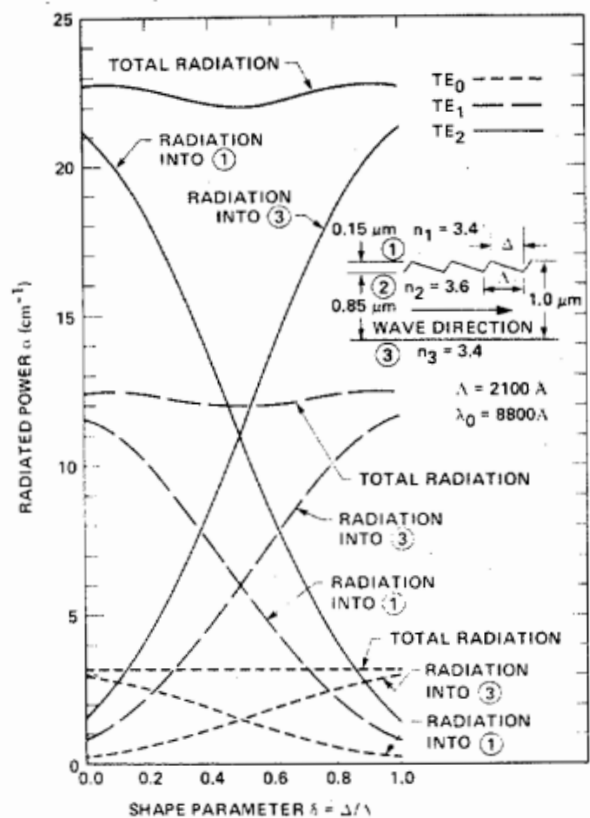

Fig. 35. The coupling loss of a waveguide triangular grating as a function of shape parameters [70].

\section{Periodic Waveguides for Nonlinear INTERACTIONS}

Periodic structures can be used for phase matching in nonlinear optical interactions [73]-[76]. The subject is too specialized to be considered in detail here. We will, however, present the basic arguments here leaving the details to the quoted references.

Consider the problem of second harmonic generation inside a waveguide from an input mode

$$
\varepsilon_{a}^{(\omega)}(x) \exp i\left(\omega t-\beta_{a}^{(\omega)} z\right)
$$

to an output mode at twice the frequency

$$
\varepsilon_{b}^{(2 \omega)}(x) \exp i\left(\omega t-\beta_{b}^{(2 \omega)} z\right) \text {; }
$$

for an appreciable conversion efficiency the phase matching condition

$$
2 \beta_{a}^{(\omega)}=\beta_{b}^{(2 \omega)}
$$

must be satisfied. If condition (105) is violated, then the interaction is limited effectively to a waveguide length of

$$
L_{c}=\frac{2 \omega}{2 \beta_{a}^{(\omega)}-\beta_{b}^{(2 \omega)}}
$$

and is usually uninterestingly small. One method of overcoming this problem is to supply the missing "momentum" by the waveguide periodicity. If the period of the spatial modulation is $\Lambda$, then phase matching is achieved provided

$$
2 \beta_{a}^{(\omega)}=\beta_{b}^{(2 \omega)}+m \frac{2 \pi}{\Lambda}
$$

for some integer $m$. The reasons for (107) are fundamentally similar to those used to derive (29). Equation (107) guaran-

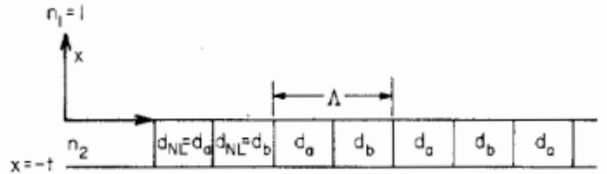

$n_{3} \quad$ Substrate

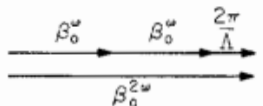

Fig. 36. A dielectric waveguide with a space periodic nonlinear property.

tees that a source polarization is produced by the spatial modulation of the fundamental mode a by the periodicity which propagates at the same phase velocity $2 \omega / \beta_{b}^{(2 \omega)}$ as that of the generated mode at $2 \omega$. The interaction is thus cumulative over the whole length of the waveguide since the coherence length $L_{c}$

$$
L_{c}=\frac{2 \pi}{2 \beta_{a}^{(\omega)}-\beta_{b}^{(2 \omega)}-m \frac{2 \pi}{\Lambda}}
$$

is infinite.

Two types of periodic perturbation have been considered. The first involves a corrugation of the waveguide [74]. It has been demonstrated recently by Chen et al. [77] .

The second method proposed [75] involves a modulation of the spatial distribution of the nonlinear material. It is potentially the more efficient of the two methods but more difficult to realize. It has not been demonstrated as yet. A possible embodiment is shown in Fig. 36.

\section{REFERENCES}

[1] J. E. Goell, "A circular harmonic computer analysis for rectangular dielectric waveguides," Bell Syst. Tech. J., vol. 48, p. 2133, 1968.

[2] E. A. J. Marcatili, "Dielectric rectangular waveguide and directional couplers for integrated optics," Bell Syst. Tech. J., vol. 48, p. $2071,1969$.

[3] P. K. Tien, R. Ulrich, and R. J. Martin, "Modes of propagating light waves in thin deposited semiconductor films," Appl. Phys. Lett., vol. 14, p. 291, 1969.

[4] J. H. Harris, R. Shubert, and J. N. Polky, "Beam coupling to films," J. Opt. Soc. Amer., vol. 60, p. 1007, 1970.

[5] A. Yariv, "Coupled-mode theory for guided-wave optics," IEEE J. Quantum Electron., vol. QE-9, p. 919, 1973.

[6] M. L. Dakss, L. Kuhn, P. F. Heidrick, and B. A. Scott, "Grating coupler for efficient excitation of optical guided waves in thin films," Appl. Phys. Lett., vol. 16, p. 523, 1970.

[7] a) F. W. Dabby, A. Kestenbaum, and U. C. Paek, "Periodic dielectric waveguides," Opt. Comm., vol. 6, p. 125, 1972.

b) H. Stoll and A. Yariv, "Coupled mode analysis of periodic dielectric waveguides," Opt. Commun., vol. 8, p. 5, 1973.

[8] H. L. Garvin, E. Garmire, S. Somekh, H. Stoll, and A. Yariv, "Ion beam micromachining of integrated optics components," Appl. Opt., vol. 12, p. 455, 1973.

[9] D. P. Shinke, R. G. Smith, E. G. Spencer, and M. F. Galvin, "Thin-film distributed-feedback lasers by ion milling," Appl. Phys. Lett., vol. 21, p. 494, 1972.

[10] W. Tsang, and S. Wang, "Simultaneous exposure and develop- 
ment technique for making gratings on positive photoresist," Appl. Phys. Lett., vol. 24, p. 196, 1974.

[11] —, "Microfabrication of two-dimensional periodic arrays by laser beam interferometric technique," Appl. Phys. Lett., vol. 27 , p. $79,1975$.

[12] J. J. Turner, B. Chen, L. Yang, J. M. Ballantyne, and C. L. Tang, "Grating for integrated optics fabricated by electron microscope," Appl. Phys. Lett., vol. 23, p. 333, 1973.

[13] C. V. Shank, and R. V. Schmidt, "Optical technique for producing $0.1 \mu$ periodic surface structures," Appl. Phys. Lett., vol. 23 , p. $154,1973$.

[14] H. W. Yen, M. Nakamura, E. Garmire, S. Somekh, and A. Yariv, "Optically pumped GaAs waveguide lasers with a fundamental $0.11 \mu$ corrugation feedback," Opt. Commun., vol. 9, p. 35, 1973.

[15] H. L. Garvin, "High resolution fabrication by ion beam sputtering," in Solid State Technology. 1973, p. 31.

[16] E. G. Spencer, and P. H. Schmidt, "Ion-beam techniques for device fabrications," J. Vac. Sci and Tech., vol. 8, p. S52, 1972.

[17] R. M. Finne, and D. L. Klein, "A water-amine complexing agent system for etching silicon," J. Electrochem. Soc., vol. 114, p. $965,1967$.

[18] Y. Tarui, Y. Kamiya, and Y. Harada, "Preferential etching and etched profile of GaAs," J. Electrochem. Soc., vol. 118, p. 118, 1971.

[19] S. Iida, and K. Ito, "Selective etching of galium-arsenide cyrstals in $\mathrm{H}_{2} \mathrm{SO}_{4}-\mathrm{H}_{2} \mathrm{O}_{2}-\mathrm{H}_{2} \mathrm{O}$ system," J. Electrochem. Soc., vol. 118, p. 768,1971 .

[20] L. Comerford, and P. Zory, "Selectively etched diffraction gratings in GaAs," Appl. Phys. Lett., vol. 25, p. 208, 1974.

[21] M. Nakamura, et al., "GaAs-GaAlAs double-heterostructure injection lasers with distributed feedback," IEEE J. Quantum Electron., vol. QE-11, p. 436, 1975.

[22] W. Tsang, and S. Wang, "Profile and groove-depth control in GaAs diffraction gratings fabricated by preferential chemical etching in $\mathrm{H}_{2} \mathrm{SO}_{4}-\mathrm{H}_{2} \mathrm{O}_{2}-\mathrm{H}_{2} \mathrm{O}$ system," Appl. Phys. Lett., vol. 28, p. 44,1976 .

[23] D. C. Flanders, H. Kogelnik, R. V. Schmidt, and C. V. Shank, "Grating filters for thin film optical waveguides," Appl. Phys. Lett., vol. 24, p. 194, 1974.

[24] A. Yariv and H. W. Yen, "Bragg amplification and oscillation in periodic media," Opt. Commun., vol. 10, p. 120, 1974.

[25] S. Wang, "Principles of distributed feedback and distributed Bragg-reflector lasers," IEEE J. Quantum Electron., vol. QE-10, p. 413,1974

[26] R. Shurbert, "Theory of optical-waveguide distributed lasers with nonuniform gain and coupling," J. Appl. Phys., vol. 45, p. 209, 1974.

[27] S. R. Chinn, "Effects of mirror reflectivity in a distributed feedback laser," IEEE J. Quantum Electron., vol. QE-9, p. 574, 1973.

[28] W. Streifer, R. D. Burnham, and D. R. Scifers, "Effect of external reflectors on longitudinal modes of distributed feedback lasers," IEEE J. Quantum Electron., vol. QE-11, p. 154, 1975.

[29] M. Nakamura and A. Yariv, "Analysis of the threshold of doubleheterojunction GaAs-GaAlAs lasers with a corrugated interface," Opt. Commun., vol. 11, p. 18, 1974.

[30] H. Kogelnik and C. V. Shank, "Stimulated emission in a periodic structure," Appl. Phys. Lett., vol. 18, p. 152, 1971.

[31] C. V. Shank, J. E. Bjorkholm, and H. Kogelnik, "Tunable distributed-feedback dye laser," Appl. Phys. Lett., vol. 18, p. 395, 1971.

[32] J. E. Bjorkholm and C. V. Shank, "Distributed-feedback lasers in thin film optical waveguides," IEEE J. Quantum Electron., vol. QE-8, p. 833, 1972.

[33] K. O. Hill and A. Watanabe, "A distributed-feedback side-coupled laser," Opt. Commun., vol. 5, p. 389, 1972.

[34] J. E. Bjorkholm and C. V. Shank, "Higher-order distributed feedback laser," Appl. Phys. Lett., vol. 20, p. 306, 1972.

[35] R. L. Fork, K. R. German, and E. A. Chandross, "Photodimer distributed feedback laser," Appl. Phys. Lett., vol. 20, p. 139, 1972.

[36] P. Zory, "Laser oscillation in leaky corrugated optical waveguide," Appl. Phys. Lett., vol. 22, p. 125, 1973.

[37] K. O. Hill and A. Watanabe, "Passive-core corrugated-waveguide laser," Appl. Opt., vol. 12, p. 430, 1973.

[38] Y. Aoyagi and S. Namba, "Laser oscillation in simple corrugated optical waveguide," Appl. Phys. Lett., vol. 24, p. 537, 1974.
[39] M. Nakamura, A. Yariv, H. W. Yen, S. Somekh, and H. L. Garvin, "Optically pumped GaAs surface laser with corrugation feedback," Appl. Phys. Lett., vol. 22, p. 515, 1973.

[40] M. Nakamura, et al., "Laser oscillation in epitaxial GaAs waveguides with corrugation feedback," Appl. Phys. Lett., vol. 23, p. $224,1973$.

[41] C. V. Shank, R. V. Schmidt, and B. I. Miller, "Double heterostructure GaAs distributed-feedback laser," Appl. Phys. Lett., vol. 25 , p. $200,1974$.

[42] D. R. Scifres, R. D. Burnham, and W. Streifer, "Distributed feedback single heterojunction GaAs diode laser," Appl. Phys. Lett., vol. 25 , p. $203,1974$.

[43] R. D. Burnham, D. R. Scifres, and W. Streifer, "Single-heterostructure distributed feedback GaAs diode lasers," IEEE J. Quantum Electron., vol. QE-11, p. 439, 1975.

[44] D. B. Anderson, R. R. August, and J. E. Coker, "Distributed feedback double-heterostructure GaAs injection laser with fundamental grating," Appl. Opt., vol. 13, p. 2742, 1974.

[45] M. Nakamura et al., "GaAs- $\mathrm{Ga}_{1-x} \mathrm{Al}_{x}$ As double heterostructure distributed-feedback diode lasers," Appl. Phys. Lett., vol. 25, p. 487,1974 .

[46] M. Nakamura, et al. "GaAs-GaAlAs double-heterostructure injection lasers with distributed feedback," IEEE J. Quantum Electron., vol. QE-11,p. 436, 1975.

[47] J. Zoroofchi and J. K. Butler, "Refractive index of n-type gallium arsenide," J. Appl. Phys., vol. 44, p. 3697, 1973.

[48] J. Umeda, M. Nakamura, and K. Aiki, "Room temperature operation of distributed feedback diode lasers with separate carrier and optical confinement," in Proc. 7th Conf. Solid State Devices, Tokyo, Japan, 1975.

[49] H. C. Casey, Jr., S. Somekh, and M. Ilegems, "Room temperature operation of low threshold separate confinement heterostructure injection laser with distributed feedback," Appl. Phys. Lett., vol. 27 , p. $142,1975$.

[50] M. Nakamura, K. Aiki, J. Umeda, and A. Yariv, "CW operation of distributed feedback GaAs-GaAlAs diode lasers at temperatures up to 300K," Appl. Phys. Lett., vol. 27, p. 403, 1975.

[51] K. Aiki, M. Nakamura, and J. Umeda, "Lasing characteristics of distributed-feedback GaAs-GaAlAs diode lasers with separate optical and carrier confinement," IEEE J. Quantum Electron., vol. QE-12, pp. 597-603, Oct. 1976.

[52] Zh. I. Alferov et al. "Semiconductor laser with extremely low divergence of radiation," Sov. Phys. Semiconductor, vol. 8, p. $541,1974$.

[53] R. D. Burnhan, D. R. Scifres, and W. Streifer, "Low-divergence beams from grating-coupled composite guide heterostructure GaAlAs diode lasers," Appl. Phys. Lett., vol. 26, p. 644, 1975.

[54] P. Zory and L. D. Comerford, "Grating-coupled double-heterostructure AlGaAs diode lasers," IEEE J. Quantum Electron., vol. QE-11, p. 451, 1975.

[55] D. R. Scifres, R. D. Burnham, and W. Streifer, "Highly collimated laser beams from electrically pumped $\mathrm{SH}$ GaAs/GaAlAs distributed-feedback lasers," Appl. Phys. Lett., vol. 26, p. 48, 1975.

[56] - "Output coupling and distributed feedback utilizing substrate corrugations in double-heterostructure GaAs lasers," Appl. Phys. Lett., vol. 27, p. 295, 1975.

[57] S. Wang, R. F. Cordero, and C. C. Tseng, "Analysis of distributed-feedback and distributed-Bragg-reflector laser structures by method of multiple reflectors," J. Appl. Phys., vol. 45, p. 3975, 1974.

[58] K. Iga and K. Kawabata, "Active Bragg reflector and its application to semiconductor laser," Jap. J. Appl. Phys., vol. 14, p. 427, 1975.

[59] H. W. Yen, W. Ng, I. Samid, and A. Yariv, "GaAs distributed feedback reflector lasers," Opt. Commun., vol. 17, p. 213, 1976.

[60] M. Okuda, K. Murata, and K. Oonaka, "Optimum design of a distributed Bragg-reflector laser with optical loss in the corrugated waveguide," Opt. Commun., vol. 16, p. 30, 1976.

[61] W. Ng, H. W. Yen, A. Katzir, I. Samid, and A. Yariv, "Room temperature operation of distributed feedback Bragg-reflector lasers," in Dig. 9th Int. Conf. Quantum Electron., June 1976, Paper T. 3.

[62] F. K. Reinhart and R. A. Logan, "GaAs- $\mathrm{Al}_{x} \mathrm{Ga}_{1-x} \mathrm{As}$ injection lasers with distributed Bragg reflectors," Appl. Phys. Lett., vol. 27, p. $45,1975$.

[63] W. Tsang and $\mathrm{S}$. Wong, "GaAs- $\mathrm{Ga}_{1-x} \mathrm{Al}_{x} \mathrm{As}$ double heterostruc- 
ture injection lasers with distributed bragg reflectors," Appl. Phys. Lett., vol. 28, p. 496, 1976.

[64] J. H. Harris, R. K. Winn, and D. G. Dalgoutte, "Theory and design of periodic couplers," Appl. Opt., vol. 11, p. 2234, 1972.

[65] M. Neviere, R. Petit, and M. Cadilhac, "About the theory of optical grating coupler-waveguide systems," Opt. Commun., vol. 8 , p. $113,1973$.

[66] K. Ogawa and W. S. C. Chang, "Analysis of holographic thin film grating coupler," Appl. Opt., vol. 12, p. 2167, 1973.

[67] S. T. Peng, T. Tamir, and H. L. Bertoni, "Leaky-wave analysis of optical periodic couplers," Electron. Lett., vol. 9, p. 150, 1973.

[68] C. C. Ghizoni, B. U. Chen, and C. L. Tang, "Theory and experiment on grating couplers for thin film waveguides," IEEE $J$. Quantum Electron., vol. QE-12, p. 69, 1976.

[69] W. Streifer, D. R. Scifres, and R. D. Burnham, "Analysis of grating-coupled radiation in GaAs: GaAlAs lasers and waveguides," IEEE J. Quantum Electron., vol. QE-12, p. 422, 1976.

[70] W. Streifer, R. D. Burnham, and D. R. Scifres, "Analysis of grating-coupled radiation in GaAs: GaAlAs lasers and waveguides-II: Blazing effects," IEEE J. Quantum Electron., vol.
QE-12, p. 494, 1976.

[71] K. Sakuda and A. Yariv, "Analysis of optical propagation in a corrugated dielectric waveguide," Opt. Commun., vol. 8, p. 4, 1973.

[72] Y. Aoyagi and S. Namba, "Blazing of holographic grating by ion etching technique," Jap. J. Appl. Phys., vol. 15, p. 721, 1976.

[73] N. Bloembergen and A. J. Sievers, Appl. Phys. Lett., vol. 17, p. 483,1970 .

[74] S. Somekh and A. Yariv, "Phase matchable nonlinear optical interaction in periodic thin films," Appl. Phys. Lett., vol. 21, p. $140,1972$.

[75] —-, "Phase matching by periodic modulation of the nonlinear optical properties," Opt. Commun., vol. 6, p. 301, 1972.

[76] C. L. Tang and P. P. Bey, "Phase matching in second harmonic generation using artificial periodic structures," IEEE J. Quantum Electron., vol. QE-9, p. 9, 1973.

[77] B. U. Chen, C. C. Ghizoni, and C. L. Tang, "Phase matched second-harmonic generation in solid thin films using modulation of the nonlinear susceptibilities," Appl. Phys. Lett., vol. 28, p. $651,1976$.

\title{
Monolithic Laser/Waveguide Coupling by Evanescent Fields
}

\author{
J. C. CAMPBELL, MEMBER, IEEE, AND D. W. BELLAVANCE
}

\begin{abstract}
Evanescent field coupling without phase matching between a double heterostructure, monolithic laser and an "external" waveguide is reported. Coupling efficiencies as high as 25 percent have been obtained.
\end{abstract}

$\mathrm{O}$ $\mathrm{NE}$ of the key problems in the development of integrated optical circuits is that of efficiently coupling light from a monolithic laser source to a waveguide circuit. Three coupling schemes have previously been reported: 1) endfire coupling [1], [2] ; 2) taper coupling [3], [4]; and 3) phase-matched coupling $[5],[6]$. Devices using endfire coupling have passive waveguides coplanar with the laser cavity. An alternate approach utilizes an intracavity taper to couple light from the laser to an underlying waveguide. A disadvantage of these coupling schemes is that they require the utmost control of the

Manuscript received September 25, 1976; revised November 12, 1977. This work was supported in part by the U.S. Office of Naval Research.

J. C. Campbell was with the Central Research Laboratory, Texas Instruments, Incorporated, Dallas, TX 75222. He is now with Bell Laboratories, Holmdel, NJ 07733.

D. W. Bellavance is with the Central Research Laboratory, Texas Instruments Incorporated, Dallas, TX 75222. growth conditions [2], [4] or a two-stage growth procedure. A less complicated structure, from a fabrication point of view, has been proposed by Watts [5] and fabricated by Suematsu et al. [6], [7]. In these lasers the coupling between the laser cavity and an external passive waveguide is analogous to that of a directional coupler. To obtain efficient coupling, the propagation constants of the laser cavity and the passive waveguide must be closely matched. This imposes severe limitations on the dimensions and compositions of the layers. In this paper we describe a device similar to the structure reported by Suematsu et al., consisting of a monolithic laser coupled to a passive waveguide. The coupling mechanism differs, however, in that the modes of the waveguide are excited directly by the evanescent fields of radiation in the laser cavity without phase matching.

The schematic in Fig. 1 shows that the laser/waveguide structure consists of five layers. Typical values for the Al concentration and thickness of each layer are indicated in the figure. The top three layers are typical of those commonly found in ( $\mathrm{Al}, \mathrm{Ga}) \mathrm{As} / \mathrm{GaAs}$ heterojunction lasers. The fourth layer has a dual function; it serves as the n-type heterobarrier as well as being the "external" waveguide. Radiation in the 